\title{
A rapid biodiversity assessment of Lesotho's first proposed Biosphere Reserve: a case study of Bokong Nature Reserve and Tšehlanyane National Park
}

\section{Authors}

1,2Lerato Seleteng-Kose (1) ${ }^{3}$ Khotso Kobisi (10

${ }^{4}$ Ruida Pool-Stanvliet (D)

${ }^{5}$ Khotso Mohap

\section{Affiliations}

'Department of Biology, National University of Lesotho, Roma 180, Lesotho.

${ }^{2}$ Department of Botany and Plant Biotechnology, University of Johannesburg, P.O. Box 524, Auckland Park 2006 , Johannesburg, South Africa. ${ }^{3}$ Independent Researcher and Plant Collector, P.O. Box 148 Machache 130, Lesotho.

${ }^{4}$ P.O. Box 6147, Uniedal, 7612, South Africa.

${ }^{5}$ Department of Environment, Ministry of Tourism, Environment and Culture, P. O Box 10993, Maseru, Lesotho

\section{Corresponding Author}

Lerato Seleteng-Kose; e-mail: leratoseletengkose@gmail.com

\section{Dates}

Submitted: 27 March 2020 Accepted: 13 May 2021 Published: 23 August 2021

\section{How to cite this article:}

Seleteng-Kose, L., Kobisi, K., PoolStanvliet, R. \& Mohapi, K., 2021 'A rapid biodiversity assessment of Lesotho's first proposed Biosphere Reserve: a case study of Bokong Nature Reserve and Tšehlanyane National Park', Bothalia 51(2), a6. http://dx.doi. org/10.38201/btha.abc.v51.i2.6

Copyright: @ 2021. The Authors Licensee: SANBI. This work is licensed under the Creative Commons Attribution 4.0 International License.
Background: Two of Lesotho's protected areas, namely Bokong Nature Reserve and Tšehlanyane National Park, form the core area of the country's first proposed Biosphere Reserve. Biodiversity is a key aspect needed to justify nomination of a Biosphere Reserve under UNESCO's Man and the Biosphere Programme. Previously documented biodiversity of the two protected areas is limited in terms of coverage and scope as well as being outdated. The aim of the current study was to conduct a rapid assessment of the biodiversity, including endemism, of the proposed Biosphere Reserve to inform the formal nomination process.

Methods: A field survey was undertaken over 112033 ha in the core, buffer and transition zones of the proposed Biosphere Reserve during which species of both flora and fauna were documented. Purposeful recordings were made during different seasons to incorporate various flowering seasons of the plants, as well as faunal species that may hibernate or migrate seasonally.

Results: A total of 380 plant species was recorded, 30 of which are legally protected in the country, 60 endemic to the Drakensberg Mountain Centre, and two species (Aloe polyphylla and Glumicalyx lesuticus) are endemic to Lesotho. The former is the national flower of Lesotho and is under threat due to illegal trade. Sixteen mammal species were recorded, seven of which are legally protected in the country, as well as 53 bird species (including the IUCN Red Listed vulture species, Gyps coprotheres and Gypaetus barbatus). Two fish species were also recorded including Pseudobarbus quathlambae, which is Lesotho's only known endemic vertebrate species, as well as seven reptile and three amphibian species (two of which are near endemic namely Amietia delalandii and $A$. vertebralis).

Conclusion: This survey has provided valuable baseline information on the biodiversity (particularly regarding the flora and avifauna) of the proposed Biosphere Reserve, which includes two protected areas namely Bokong Nature Reserve and Tšehlanyane National Park. The findings reflect the biodiversity value of the area and will contribute towards its nomination as Lesotho's first Biosphere Reserve.

Keywords: Drakensberg Mountain Centre, MAB Programme, endemics, Red Listed species, UNESCO

\section{Introduction}

The Man and the Biosphere (MAB) Programme, of the United Nations Educational, Scientific and Cultural Organization (UNESCO), is an intergovernmental scientific programme that seeks to establish a scientific basis for the improvement of relationships between people and their environments. The Programme is implemented through establishment of Biosphere Reserves (BRs), which are 
aimed at balancing human's dependence on biological resources with the ecological integrity of the natural resource base. The MAB Programme contributes to implementing elements of the Convention on Biological Diversity (CBD) through i) conservation of genetic resources, species and ecosystems; ii) scientific research and monitoring; and iii) promoting sustainable development in communities (UNESCO 1996). The guidelines indicate that each BR should contain three elements, namely: a core area/s, which is/are securely protected for conserving biological diversity as well as undertaking non-destructive research and other low-impact uses; a buffer zone, which usually surrounds or adjoins the core area/s, and is used for co-operative activities compatible with sound ecological practices; a flexible transition area, which incorporates a variety of activities including agriculture and human settlements where various stakeholders work together to manage and sustainably develop the area's resources (UNESCO 1996).

In Lesotho, two protected areas (PAs) namely Bokong Nature Reserve (BNR) and Tšehlanyane National Park (TNP) form the core areas of a proposed BR (Figure 1). The area occurs within the Grassland Biome of southern Africa, which is the second largest biome after the Savanna Biome (Mucina \& Rutherford 2006). The PAs have been established under the auspices of the Lesotho Highlands Development Authority (LHDA). The BNR covers an area of about 1970 ha, and is endowed with wetlands that provide water to the Katse Dam, which in turn supplies water to Gauteng Province in South Africa (LHDA 1998a). The wetlands also provide a habitat to the rare Sloggett's ice rat (Otomys sloggetti Thomas, 1902), which is endemic to southern Africa, being confined to South Africa and Lesotho (Maloti-Drakensberg).

Interestingly, BNR is the highest elevation PA in Lesotho, lying between 2600 and $3152 \mathrm{~m}$ above sea level (m.a.s.l). Tšehlanyane National Park covers an area of $5600 \mathrm{ha}$, and serves as a conservation area for mature Leucosidea sericea Eckl. \& Zeyh. riparian woodland, which forms the largest woodland cover of all areas in Lesotho. Woodland vegetation covers only about $1 \%$ of the whole country (May 2000). The most conspicuous plants in TNP is the oldwood (Leucosidea sericea), quilted sagewood (Buddleja salviifolia (L.) Lam. and Drakensberg bamboo (Thamnocalamus tessellatus (Nees)) (Soderstrom \& R.P.Ellis 1982). The latter is the only endemic southern African bamboo, and occurs from the Western Cape province of South Africa, through Lesotho and KwaZulu-Natal to the Free State, at elevations of about 1500-2 500 masl (Soderstrom \& Ellis 1982). Indeed, the name of the park originates from the colour of the Drakensberg bamboo, which is yellowish (translating into Tšehlanyane in Sesotho - the local language). The plant is globally rare and provides a habitat for the Red Listed, Endangered butterfly known as the Bamboo Sylph (Metisella syrinx (Trimen, 1868)), which is known only from mountain summits of South Africa (Eastern Cape, KwaZulu-Natal) and Lesotho (Soderstrom \& Ellis 1982).

Bokong Nature Reserve and TNP fall within the high elevation catchments of Lesotho (Lahmeyer Macdonald Consortium \& Oliver Shand Consortium 1986). Being situated at a high elevation, up to 3152 masl, the proposed BR experiences occasional snow, particularly during winter months (May to July). This has resulted in the area developing unique subalpine vegetation typical of the Maloti-Drakensberg (Mucina \& Rutherford 2006). It is estimated that $30 \%$ of the plant species occurring in the area are endemic (NES 2000). However, Carbutt and Edwards (2001) considered this to be an over-estimate since the flora of the Lesotho Highlands and Eastern Cape Drakensberg was poorly explored at the time, and thus was not incorporated into the calculation of the area's endemism. Nevertheless, the area is recognised as one of southern Africa's eight hot-spots of botanical diversity as indexed by its species richness and endemism (Cowling \& Hilton-Taylor 1994). Van Wyk and Smith (2001) rank the area as having the fourth richest regional flora in southern Africa.

A number of studies have discussed the biodiversity of the proposed BR, however, they were based largely on outdated literature. In addition, most of these studies were limited either in terms of scope, documenting mainly dominant and economically important species, or extent, covering only parts of the proposed BR. For example, the Conservation Development and Management Plan of BNR (LHDA 1998a) estimated floral species richness of the area at 180 flowering plants, based mainly on literature. Similarly, the Management and Development Plan for TNP (LHDA 1998b) estimated the floristic composition of the park at 220 flowering species, which is representative of the western Maloti (i.e. from Sentinel Peak to Mohale's Hoek) of the greater Drakensberg region, and documented 24 mammalian species, even though no field assessment had been made.

Insights into the biodiversity of the proposed BR were provided by Carbutt and Edwards (2004) who documented the native flora of the Drakensberg Alpine Centre (DAC), currently known as Drakensberg Mountain Centre (DMC), as comprising 2818 taxa in 205 families and 781 genera. Of the 2818 taxa, $89 \%$ were angiosperms, $7 \%$ bryophytes, $3 \%$ pteridophytes and $0.2 \%$ gymnosperms. In addition, they recorded 166 exotic angiosperm species. However, the work of Carbutt and Edwards (2004) is outdated and relied on literature, using regional floras such as Jacot Guillarmod (1971) and Kobisi and Kose (2003) for the Flora of Lesotho, Hilliard and Burtt (1987) and Meter et al. (2002) for the southern KwaZulu-Natal Drakensberg; and Porter et al. (1999) for the uKhahlamba-Drakensberg Park; as well as regional field guides for the trees (Pooley 1993) and mountain flowers (Pooley 2003) in the 


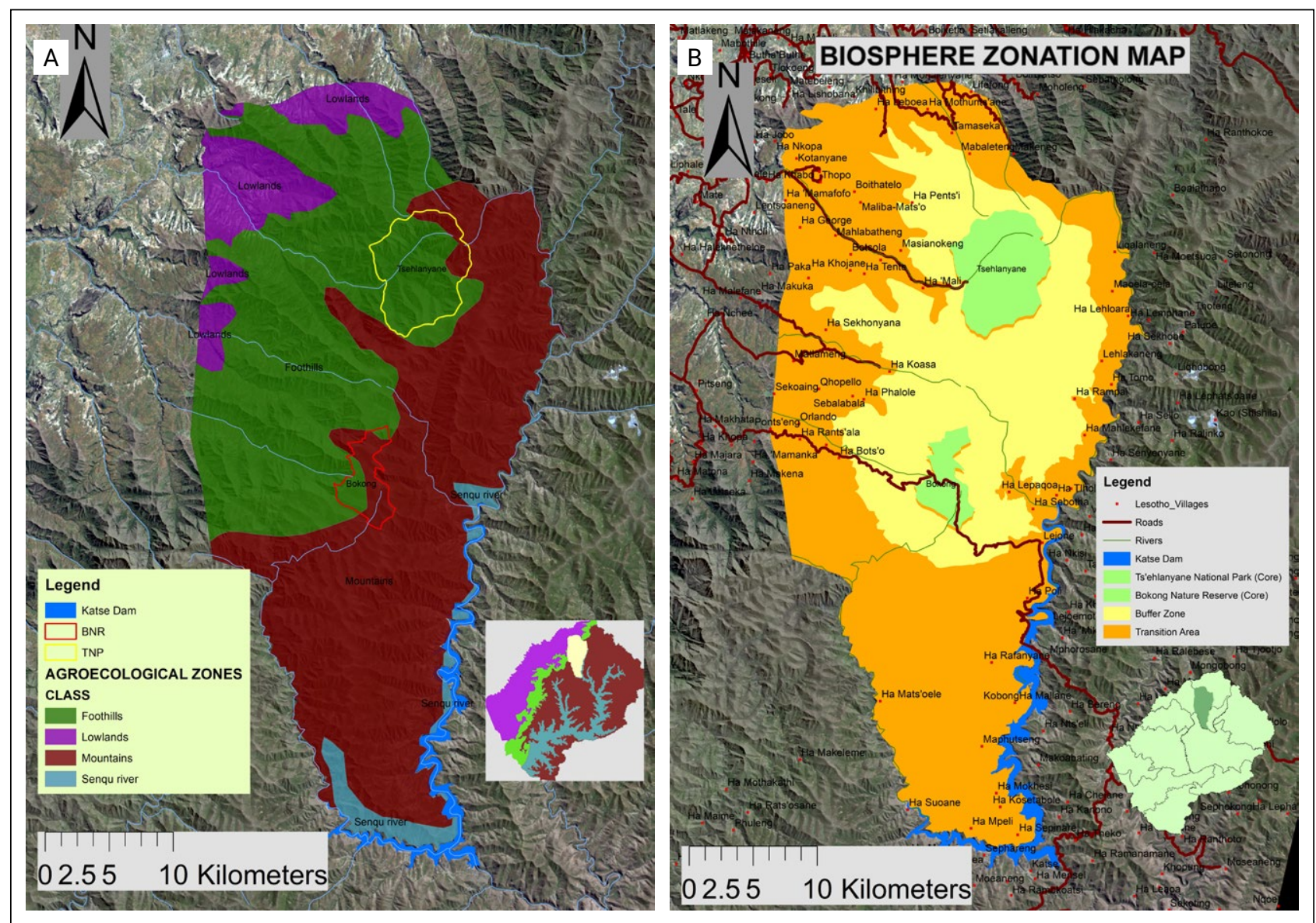

Figure 1. A \& B, Lesotho's first proposed Biosphere Reserve, showing the core (Tšehlanyane National Park and Bokong Nature Reserve), buffer and transition zones (Source: T. Leballo).

Maloti-Drakensberg. As they were using regional references, Carbutt and Edwards (2004) were not able to specify which of the recorded species occur in the PAs of the proposed BR.

A publication by Carbutt and Edwards (2006) focused on the endemic and near-endemic angiosperms of the DAC, recording 334 and 595 species respectively, also based on existing literature. The study emphasised that the flora of the area must continue to receive conservation attention because many of its taxa are rare and highly restricted in distribution, and more than half of its endemics are regarded as either Red or Orange Data species. Carbutt (2019) revised the profile of angiosperm endemics provided by Carbutt and Edwards (2006), as well as the boundary of the former DAC. The study recorded 227 angiosperm species, representing 90 genera, as endemic to the DMC, based on previous literature, as well as using Google Earth Pro to delineate new boundaries of the DMC. The revision resulted in removal of about $32 \%$ of the species, which were previously regarded as endemic from the account of Carbutt and Edwards (2006), and added two more recently described species to the list.

The most focused work on the biodiversity within the proposed BR was conducted by Letšela et al. (2003), who presented findings from a survey aimed at inventorying resources on which people rely, and that were likely to be affected by the establishment of a BR that encompases both TNP and BNR. This publication is now also outdated and was confined to the proposed core area, as well as to specific taxa with socio-economic benefit. It was therefore evident that no comprehensive documentation of the biodiversity of the proposed BR had been undertaken. As a result, a detailed biodiversity survey covering the core area (BNR and TNP) as well as buffer and transition zones, was necessary to appraise the value of the area, hence supporting its nomination as a BR.

\section{Methodology}

\section{Study area}

The proposed BR covers a total area of about $112033 \mathrm{ha}$, encompassing the core (BNR and TNP, $7570 \mathrm{ha})$, buffer (31 $050 \mathrm{ha})$ and transition zones (66 577 ha) (Figure 1), with an elevation ranging between 1636 and 3152 masl The area falls within the temperate grassland of southern Africa. It is located in the Leribe District in the northern part of Lesotho 
(Figure 1A,B). The proposed BR forms part of the larger area covered by the Maloti-Drakensberg Transfrontier Conservation and Development Programme (MDTCDP), which spans the border between Lesotho and South Africa, covering certain parts of the two countries (northeastern, eastern and southeastern parts of Lesotho, as well as the adjacent parts of South Africa).

\section{Data collection}

The survey stems from the need to profile the biodiversity of the proposed BR, which is needed for supporting nomination of the area to UNESCO. Therefore a rapid survey was undertaken, targeted at both flora and fauna occurring within the proposed BR (core, buffer and transition zones). Four purposeful surveys were conducted by the second and fourth authors, spanning different seasons between August 2018 and May 2019. This was done to target flowering seasons of different plant species to enable their identification, as well as to maximise opportunities to observe faunal species that may hibernate or migrate seasonally. Each of the four surveys lasted for a period of two weeks. Research and collecting permits were provided by the Department of Environment (under the Ministry of Tourism, Environment and Culture in Lesotho), which is also the custodian of the core area that forms part of the proposed BR.

The approach used for data collection was a purposeful field survey undertaken by travelling throughout the core, buffer and transition zones of the proposed BR on foot and on horseback, targeting accessible areas of the proposed BR. Data were collected by identifying and documenting species of both flora and fauna encountered in the area. Global Positioning System (GPS) coordinates were recorded (using Garmin Etrex 10) to map the area covered during the survey (Figure 2). Plants that could not be identified on site were collected and later identified using the literature or herbarium collections at the National University of Lesotho (NUL) herbarium (ROML), where the collected specimens were also deposited.

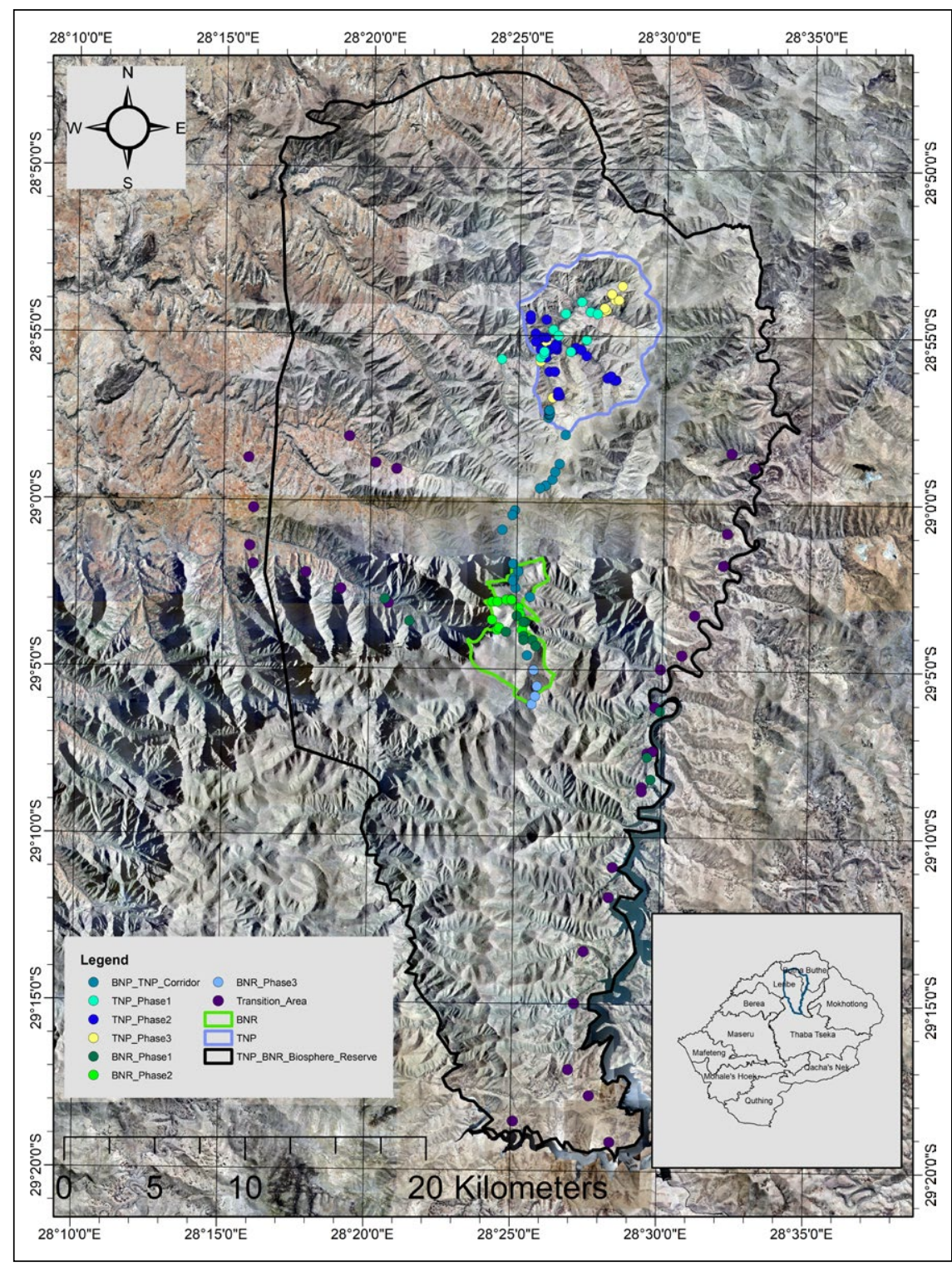

Figure 2. Transect walks covered during the different phases of the biodiversity survey (data collection) in the proposed Biosphere Reserve (Source: T. Leballo). 
Fish and aquatic amphibian species were surveyed with the aid of an electro-shocker (powered by a generator) which forced organisms to float on the water surface, where they were identified. The shocking effect was temporary to avoid causing harm. The assessment was conducted in both the Bokong and Tšehlanyane rivers.

Reptiles were surveyed by turning over stones and searching in the thicket. Birds were photographed using a Canon EOS 1100D camera (with a telephoto lens of $300 \mathrm{~mm}$ ), and identified at a later stage, whereas mammals were recorded through observation. The majority of the species were photographed to allow confirmation of their identification.

Data collection and identification of species was conducted on-site by the second author, a renowned biodiversity specialist who has contributed to various literature resources on Lesotho's flora, such as the Preliminary Checklist of the Plants of Lesotho (Kobisi 2005), a Checklist of Grasses of Lesotho (Kobisi \& Kose 2003), as well as the Grassland Biome chapter in the Vegetation of South Africa, Lesotho and Swaziland (Mucina et al. 2006). Species identification was also confirmed by the first author who is a botanist and a Senior Lecturer at the National University of Lesotho. The fourth author also formed part of the expedition team, being the Conservation Officer in the Lesotho's Northern Parks (under which BNR and TNP fall), who has also been involved in several biodiversity expeditions in the country. Various literature resources were used for identification including: Moffett (1997, 2010), Van Wyk and Van Wyk (1997), Pooley (1998, 2003), Van Oudtshoorn (1999), May (2000), Van Wyk and Gericke (2000), Talukdar (2002), Coopero-Driver et al. (2008), Van Wyk et al. (2009), Bromilow (2010), Van Wyk and Smith (2014) for plants; Stuart and Stuart (2015) for mammals; Sinclair et al. (2002) for birds; Alexander and Marais (2008) for reptiles; Du Preez and Carruthers (2009) for amphibians; and NES (2000) for fish.

\section{Limitations of the study}

The surveys were undertaken when Lesotho was experiencing dry conditions due to delayed rains in 2018 to 2019. As a result, this delayed the flowering season of the majority of the plants and some specimens could only be identified to genus level. The area is not easily accessible and surveys required long, arduous walks, lasting from sunrise to sunset and are situated in areas with mountainous topography (Figure 3A, B).

\section{Results and discussions}

\section{Species lists}

The current study provides a detailed compilation of species recorded in the proposed BR (core, buffer and transition zones), covering both flora and fauna (mammals, birds, reptiles, fish and amphibians). Species lists are provided in Tables 1-6, where scientific, English (common) and vernacular (Sesotho) names have been provided, where available. The Plantzafrica website (pza.sanbi.org), as well as Pooley $(1998,2003)$ were used as the basis for the plant names for taxa recorded in the current study. In the case of fauna, Sinclair et al. (2002) was used for birds; Stuart and Stuart (2007, 2015) for mammals; Alexander and Marais (2008) for reptiles; NES (2000) for fish; and Du Preez and Carruthers (2009) for amphibians. The IUCN Red List status of the different species is also provided, where available. Lesotho conducted the last flora Red List assessment in 2002, focusing on a limited number of species, and based on literature (Talukdar, 2002). Therefore in most cases the Red List of South African plants has been used in the current study (http://redlist. sanbi.org). Similarly, in the case of fauna, Red Listing for Lesotho was last undertaken in 2000, also based on literature. Therefore the IUCN Red List of Threatened Species website (https://www.iucnredlist.org) has been used to provide the conservation status of the fauna species listed in Tables $2-6$.

\section{Flora}

A total of 380 plant species were recorded in the proposed BR during the four biodiversity surveys undertaken (Table 1). However, six species were only identified to genus level due to absence of flowers at the time of undertaking the survey. These include Cyrtanthus sp. Aiton, Delosperma sp. N.E.Br and Eulophia sp. R.Br. ex Lindl. Thirty of the 380 recorded species are declared protected by law in the country (Legal Notice No. 36 of 1969, as amended by Legal Notice No. 93 of 2004 and No. 38 of 2006), and these include Drakensberg bamboo, giant alepidea (Alepidea amatymbica Eckl \& Zeyh.), Lesotho carnation (Dianthus basuticus Burtt Davy), quilted sagewood (Buddleja salviifolia (L.) Lam.) and Aloe L. spp. (Table 1). The most represented genera include Helichrysum Mill. consisting of 25 species, followed by Senecio L. with eight species and Aloe with six species. The findings of the current study are in agreement with those of Cowling and Hilton-Taylor (1994), who ranked Helichrysum and Senecio as the top two genera with the largest number of species in the area. Similarly, Carbutt and Edwards (2006) indicated that these two genera contribute the most to the endemic and near-endemic species of the area. In addition, LHDA (1998b) also reported extensive Drakensberg bamboo communities in the area.

Of the 380 recorded plant species, 41 are exotic (marked with an asterisk in Table 1), these include black poplar (Populus nigra L.), four o'clock (Mirabilis jalapa L.), khakiweed (Tagetes minuta L.), inkberry (Phytolacca octandra L.), peach tree (Prunus persica (L.) Batsch) and 

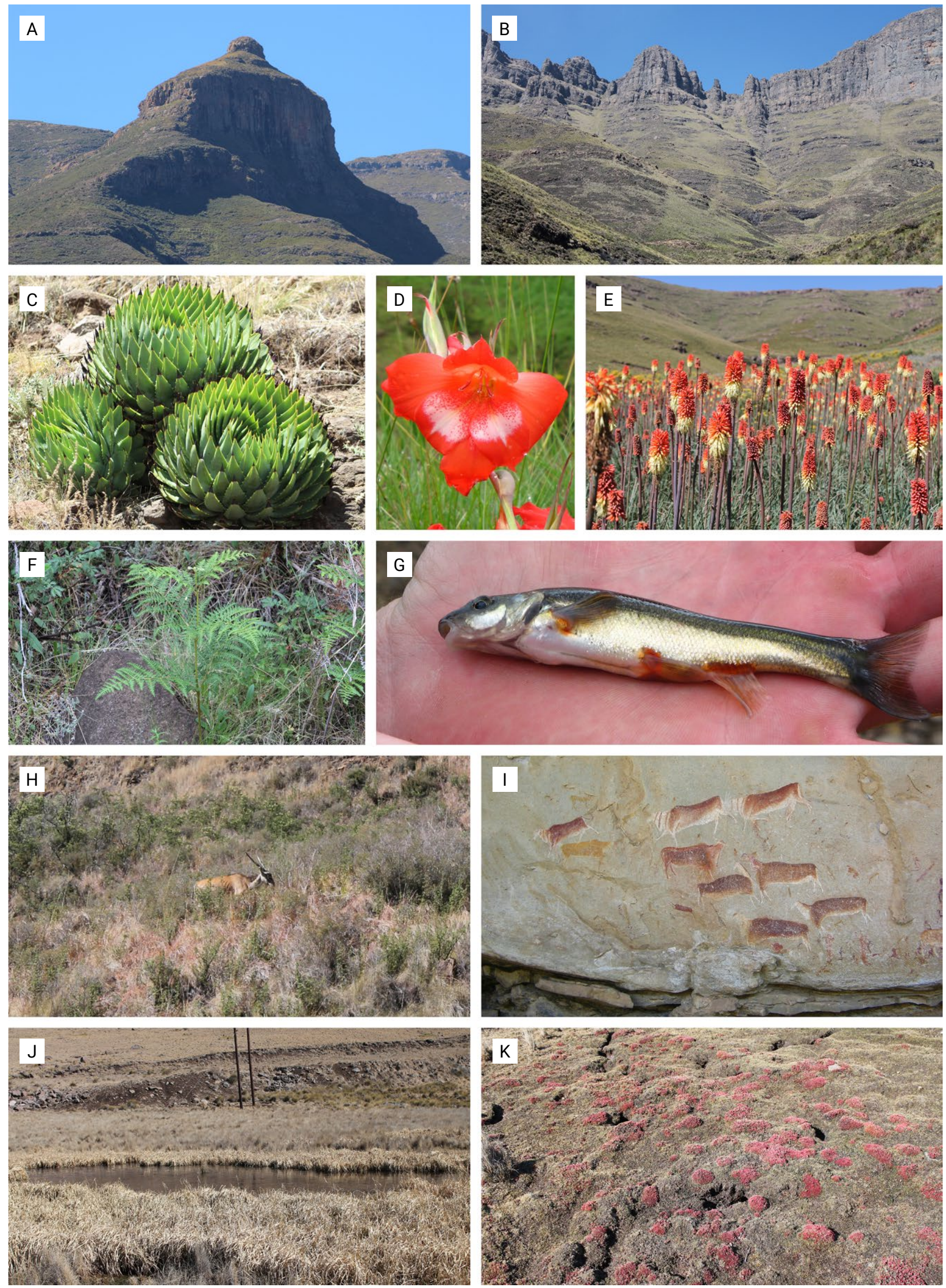

Figure 3. A \& B, the spectacular scenery of mountaineous landscapes forming part of the proposed BR; C, the endemic spiral aloe; D, the near-endemic Lesotho lily; $\mathrm{E}$, the Lesotho red-hot poker; F, locally uncommon fern - bracken; $\mathrm{G}$, endemic Maloti minnow; $\mathrm{H}$, common eland; and I, its presence on rock paintings; J, some of the wetlands in the BNR; K, some of them damaged by diggings of Sloggett's ice rat (Source: K. Kobisi). 


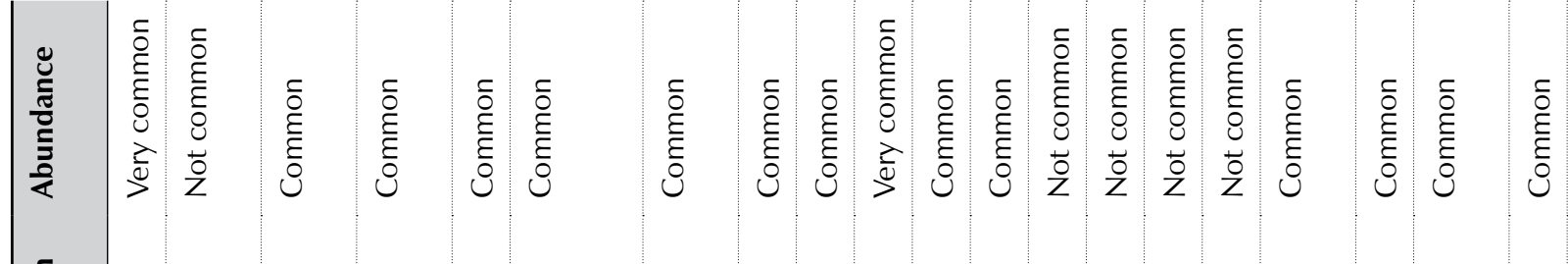

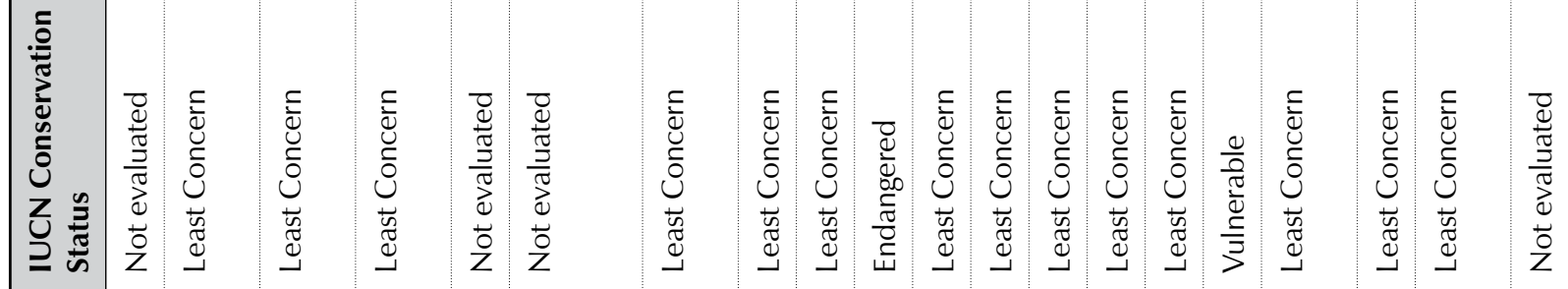

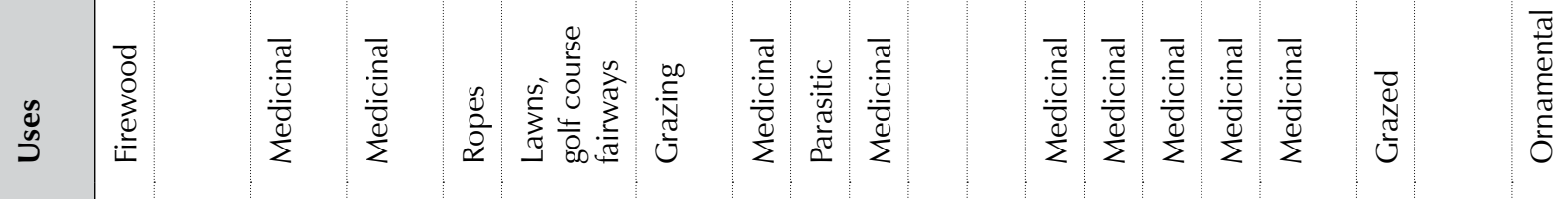

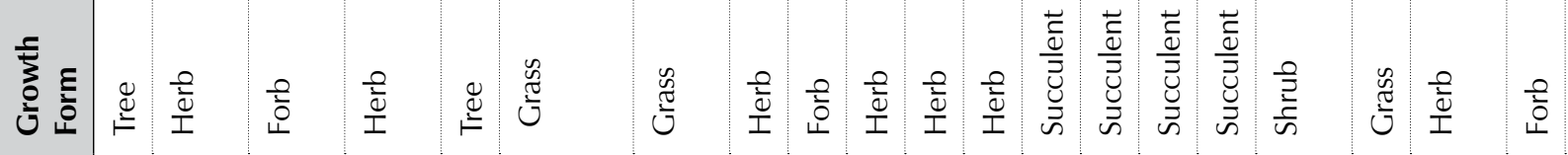

IIIIIIIIIIIII

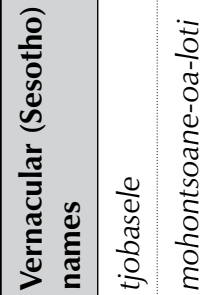

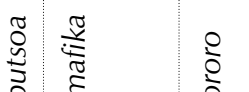

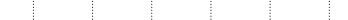

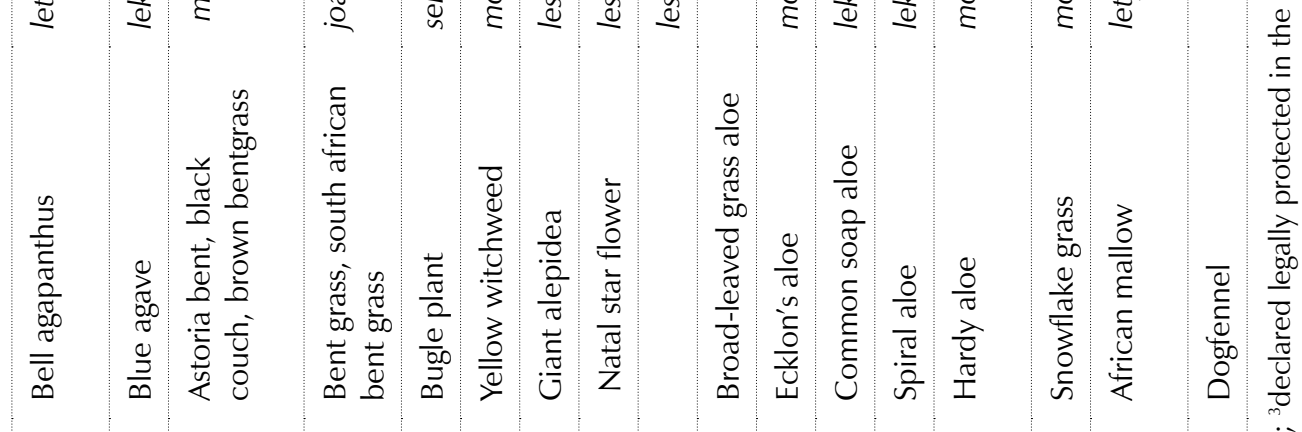




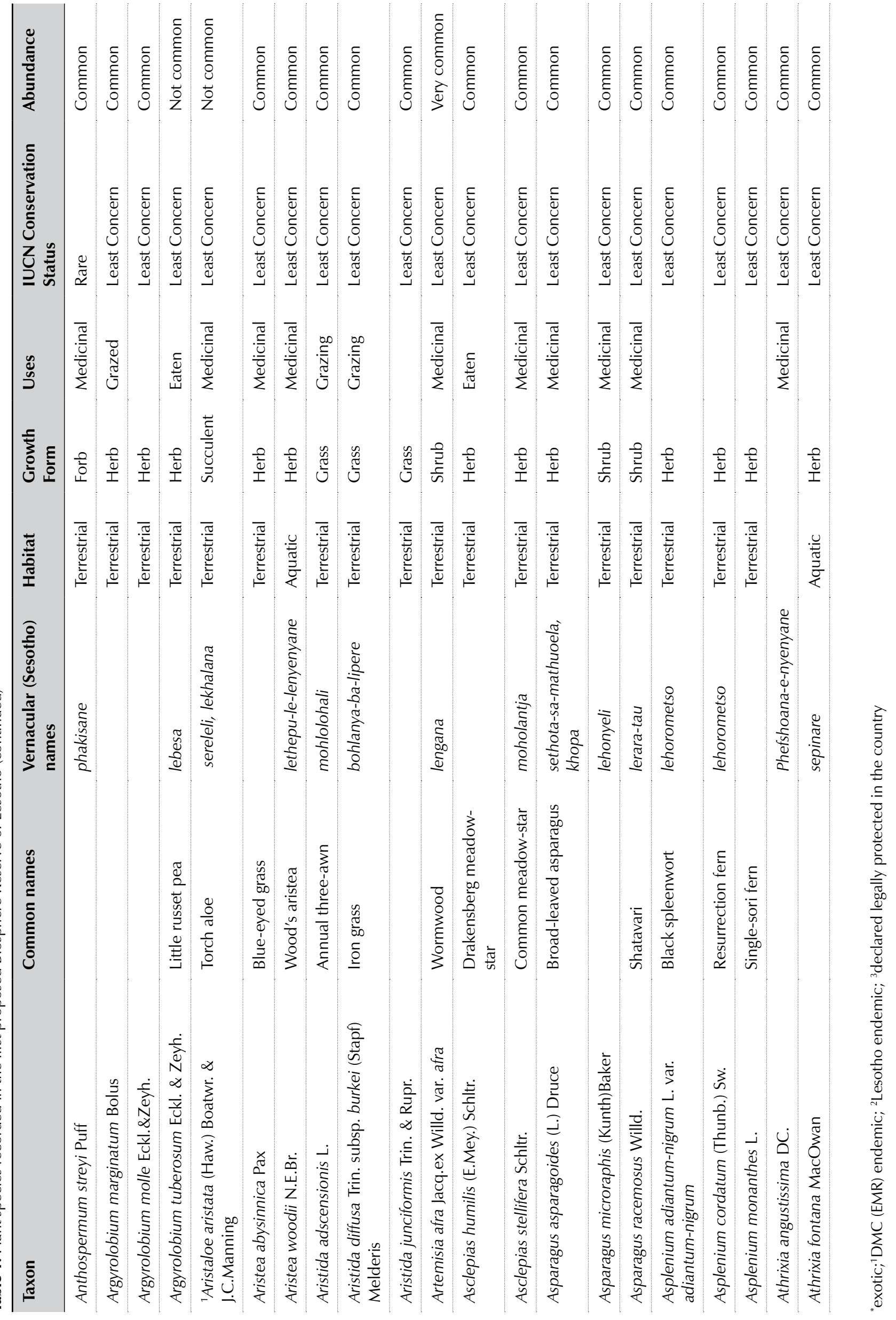




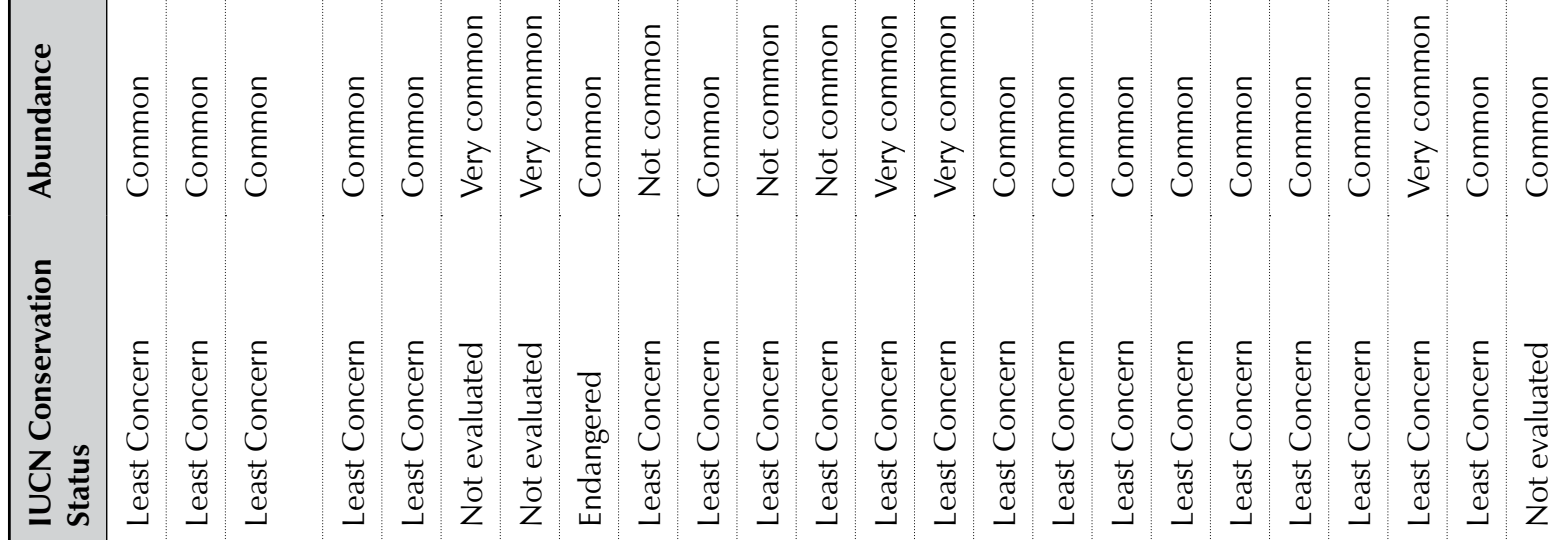

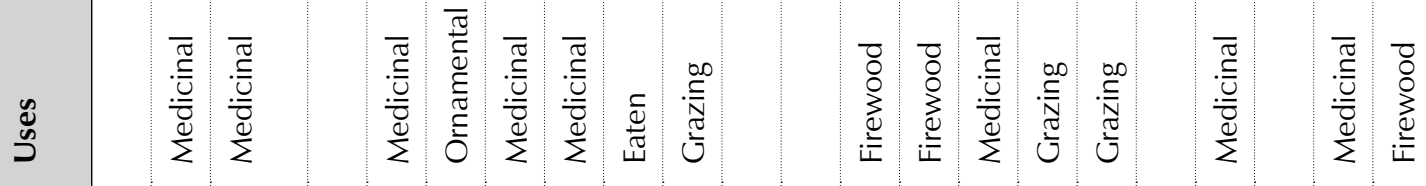

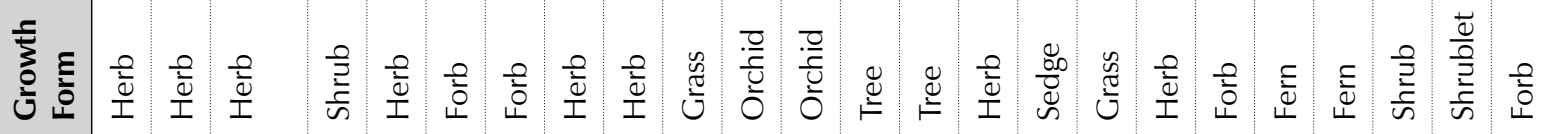

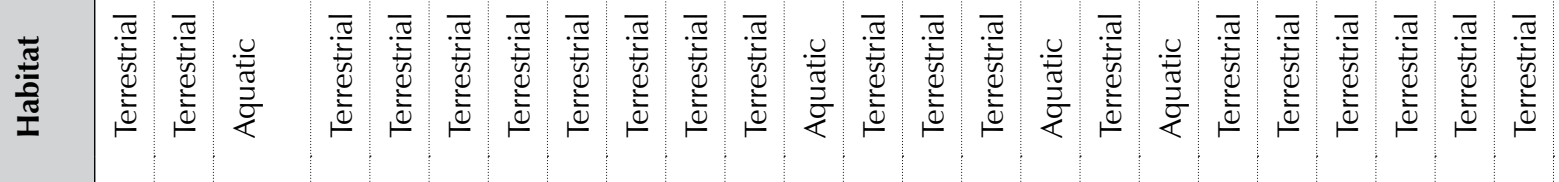

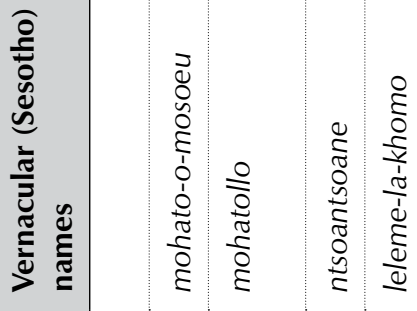

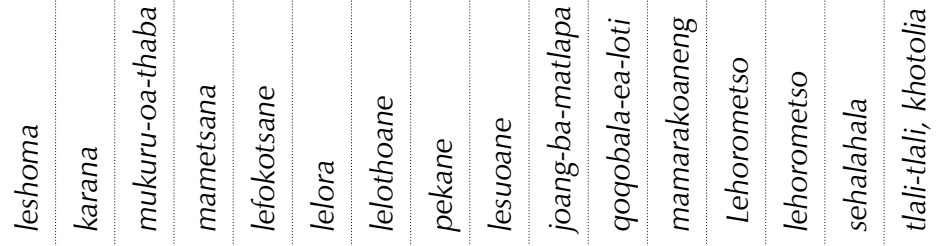

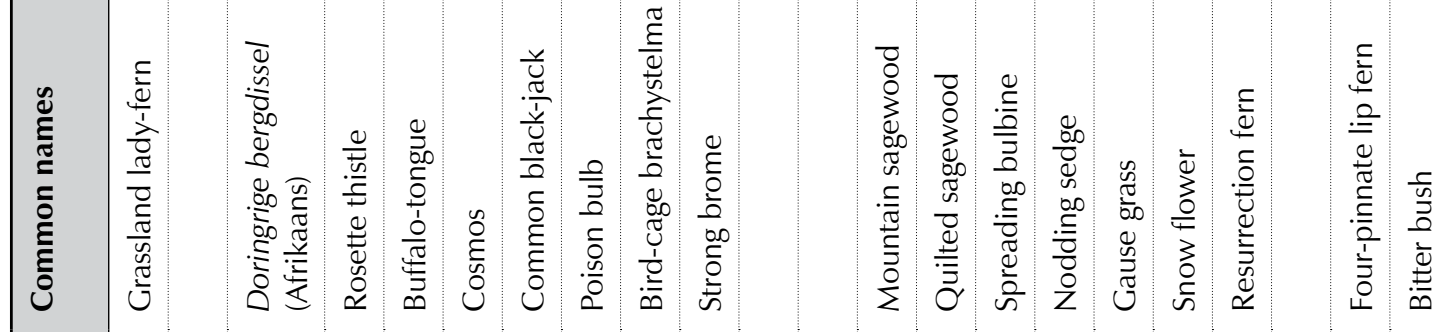

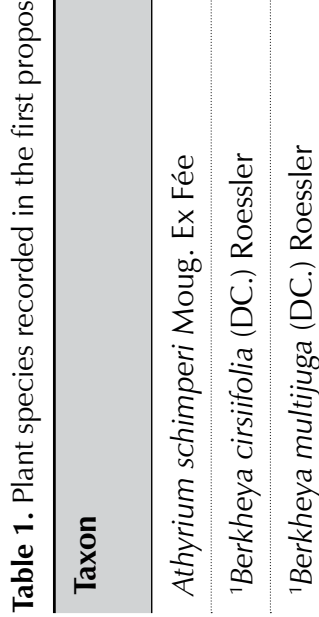

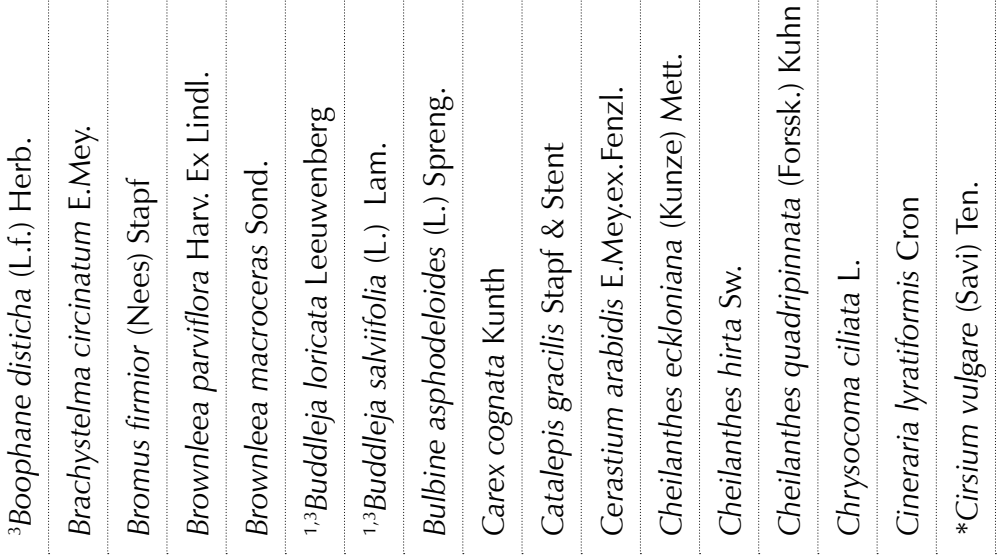




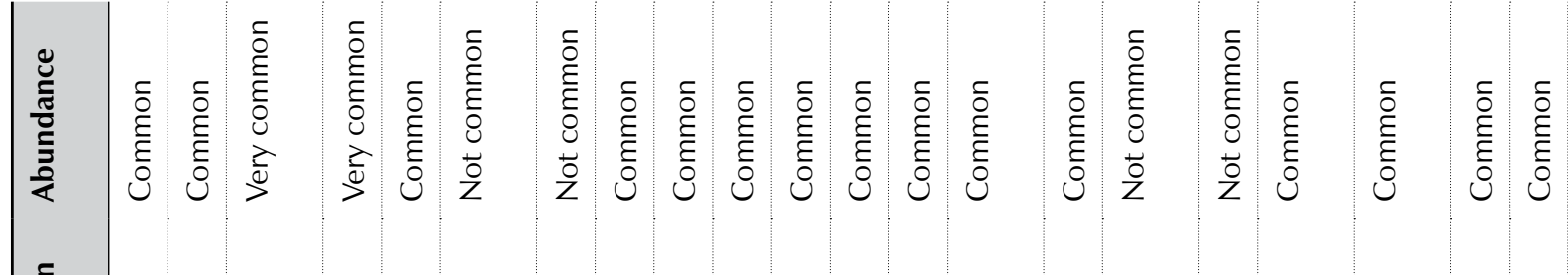

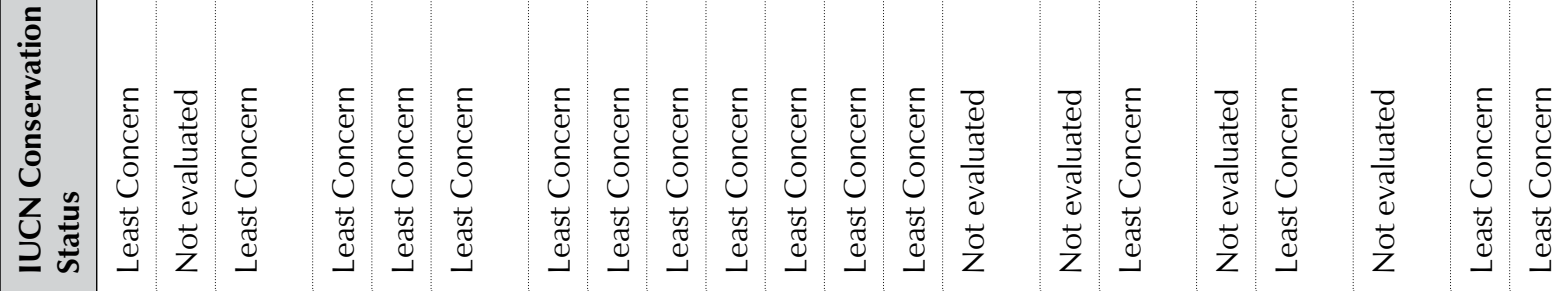

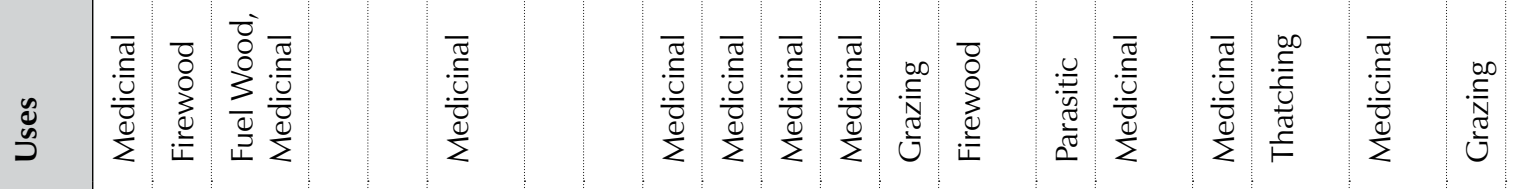

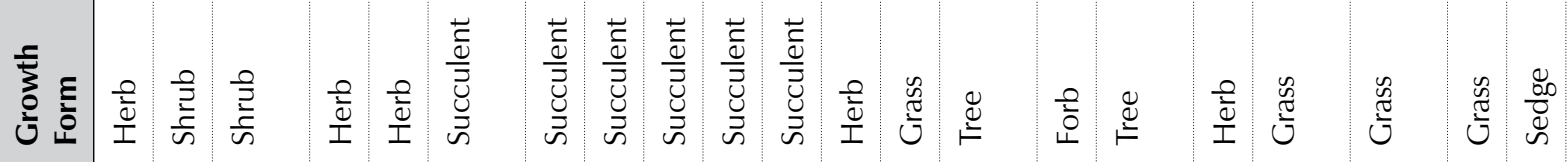

III UnIIIIIIIIIIIIIII

III

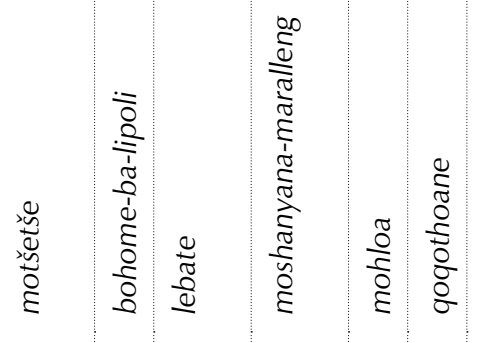

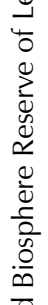
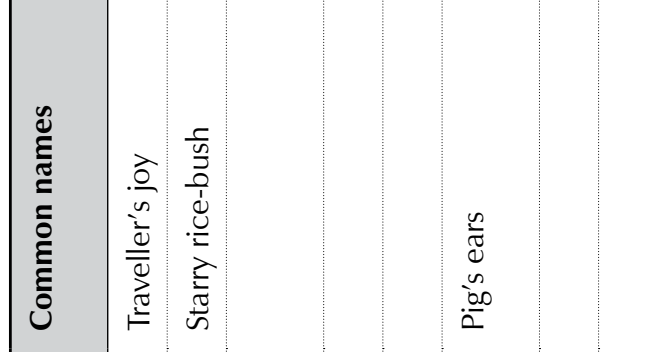

흔

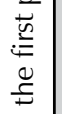

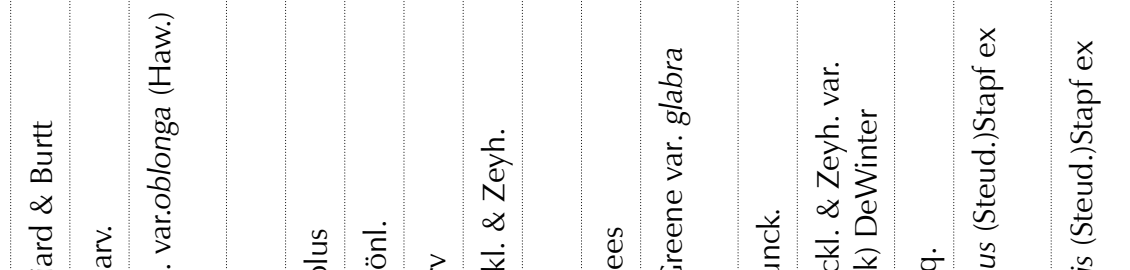

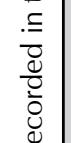

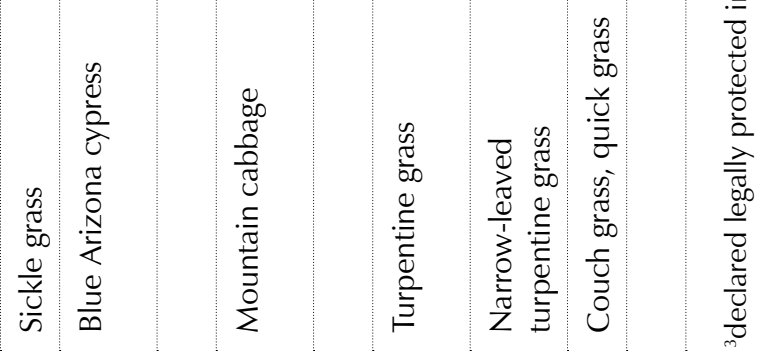

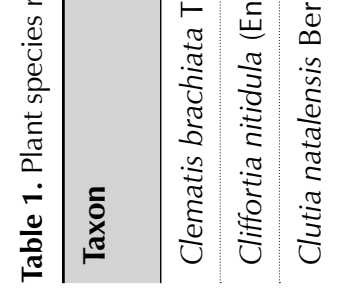

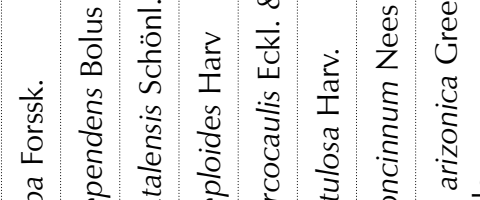

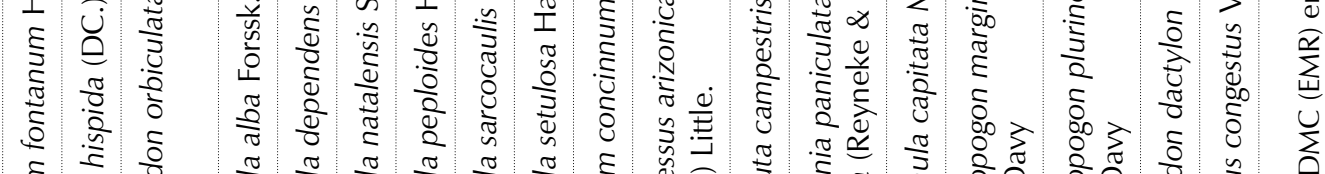

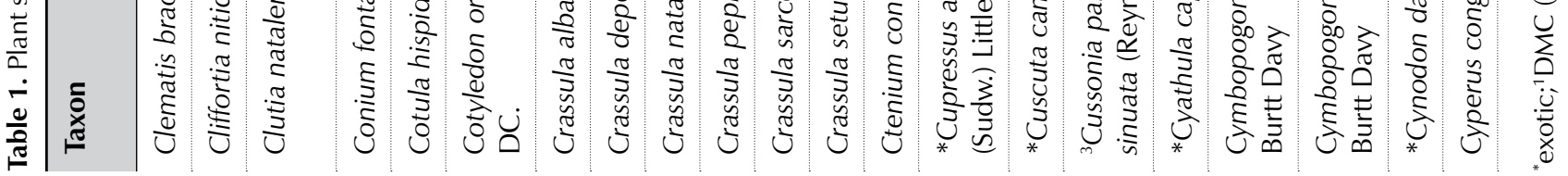




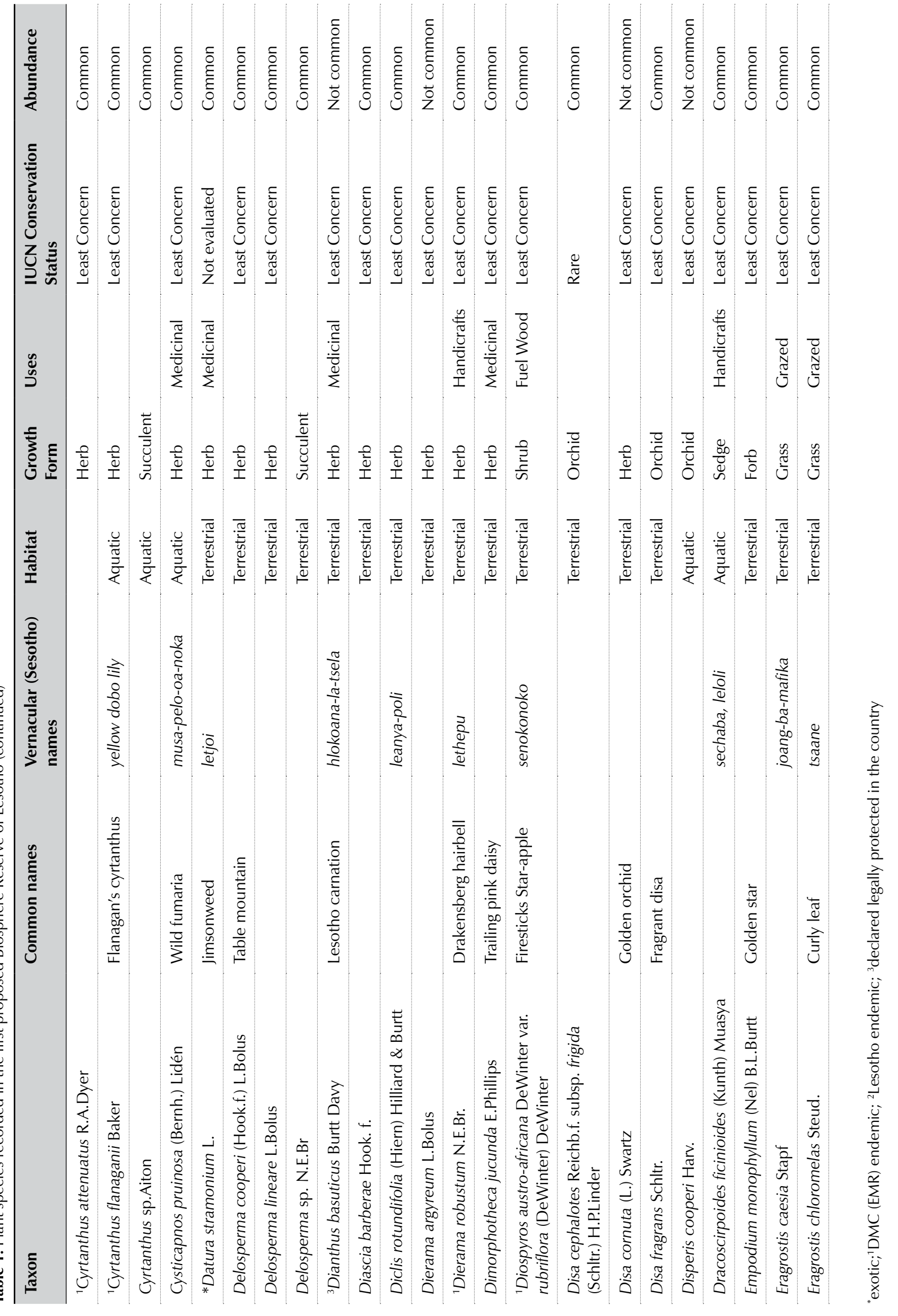




\section{HIIIUUUIUIIUUIII \\ g)

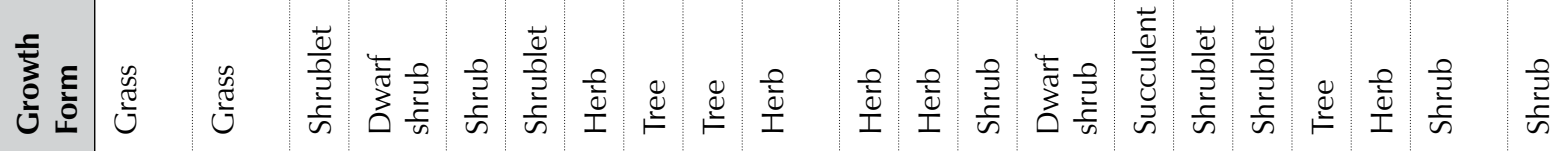

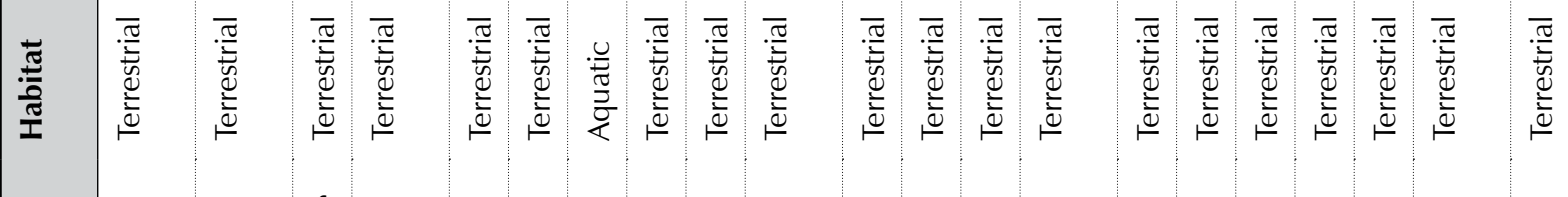

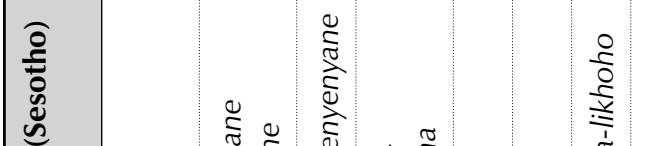

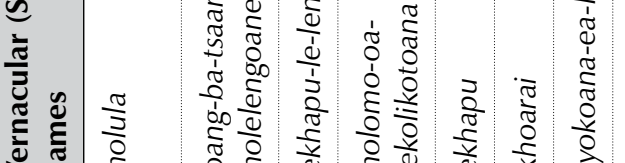

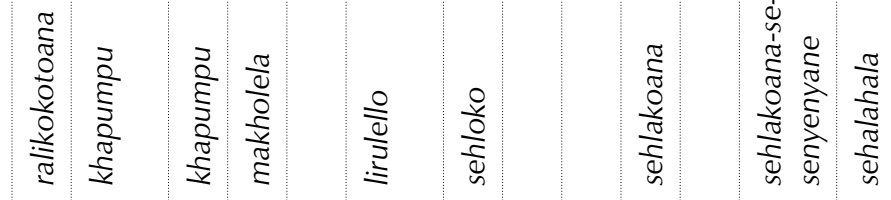

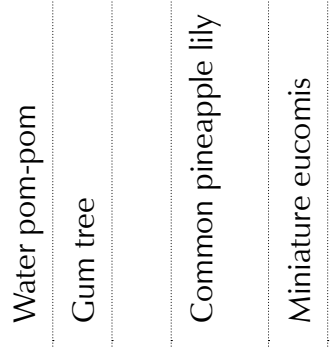

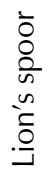

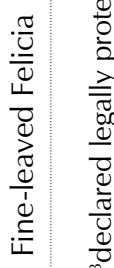

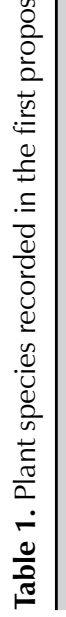

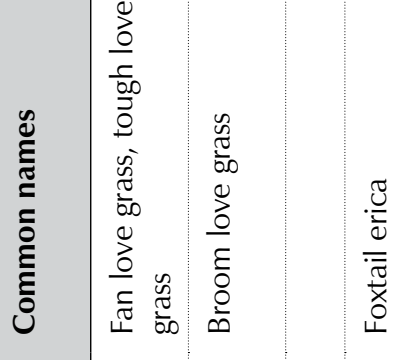

$\frac{\dot{0}}{\frac{n}{5}}$

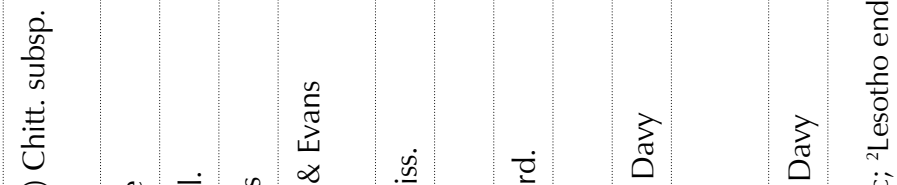

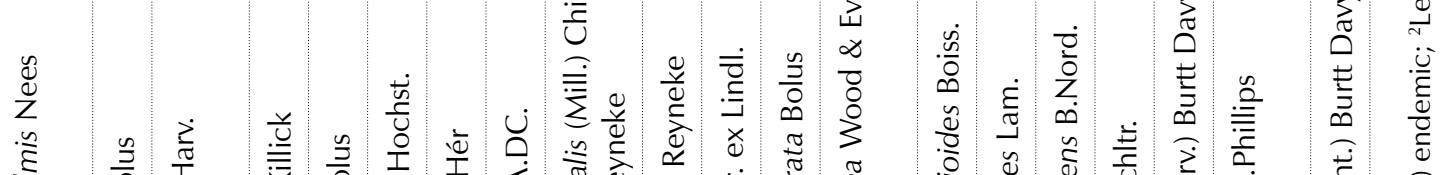

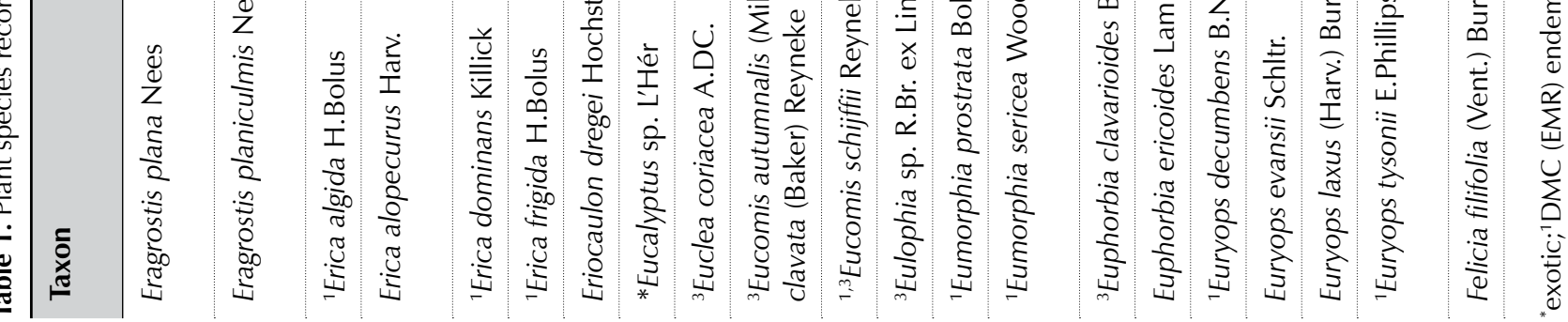




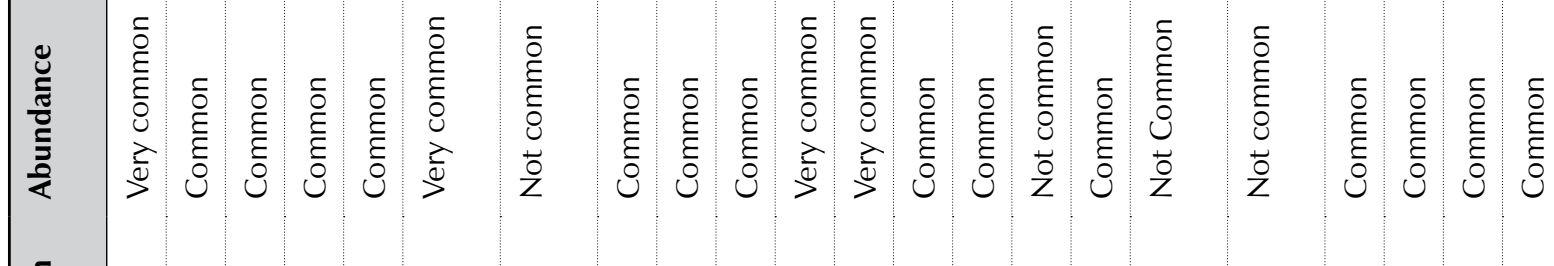 \\ 4
4
4

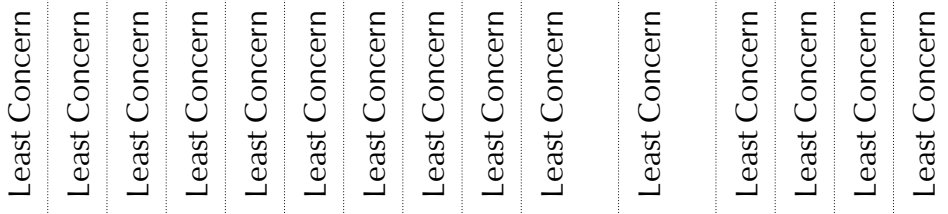

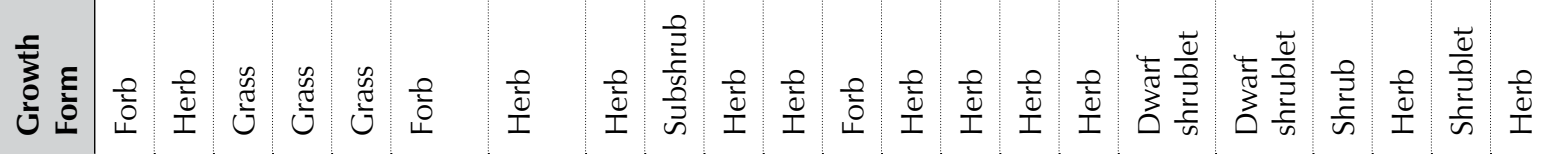

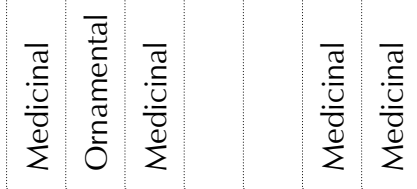

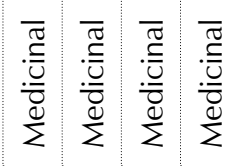

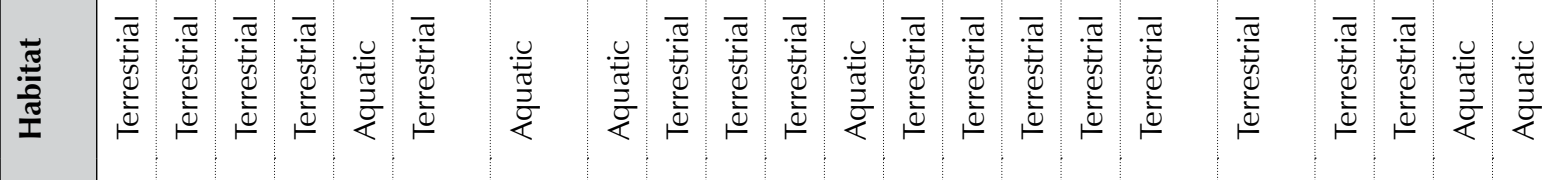

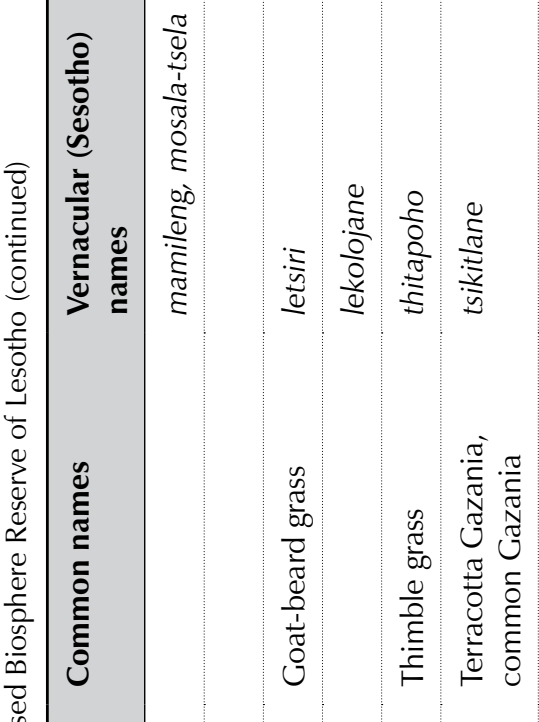




\section{MIIIIIUIIIIIIIII

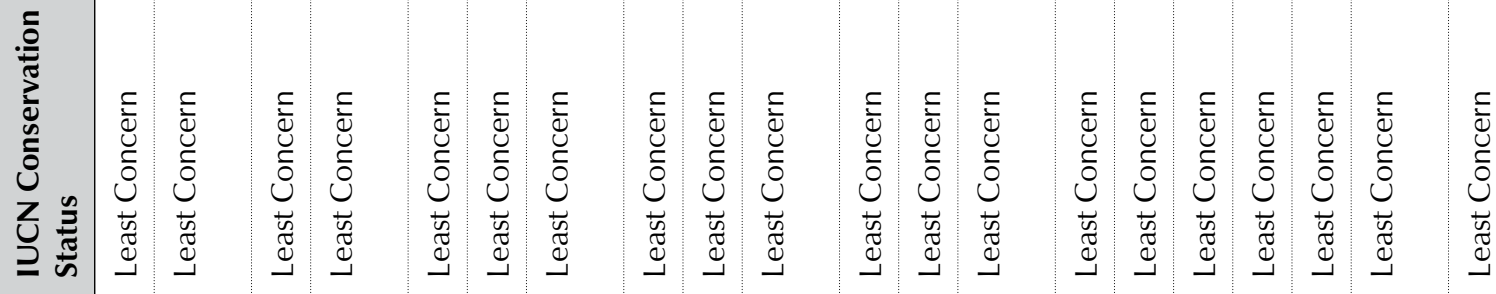

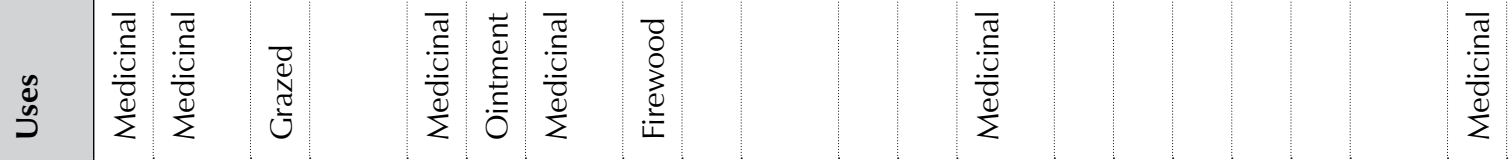

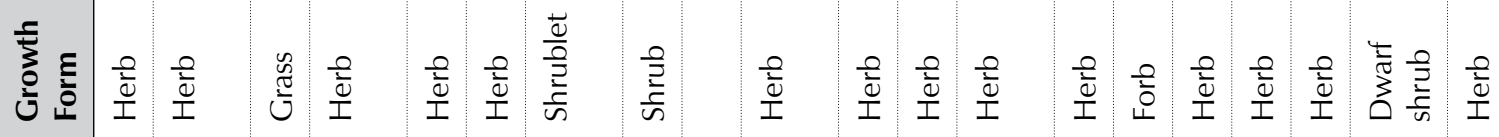

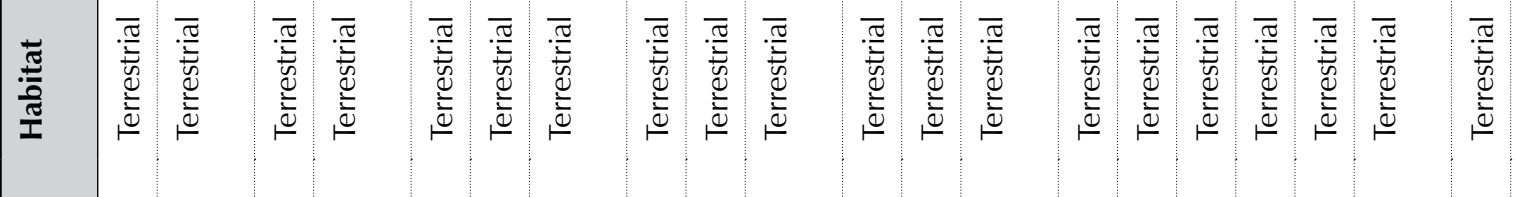

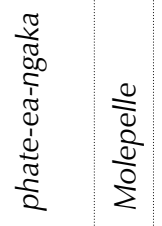

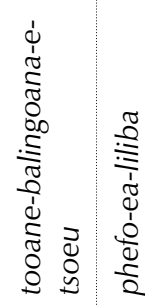

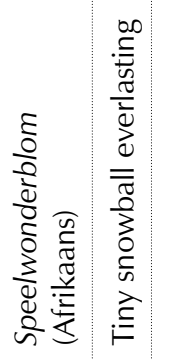




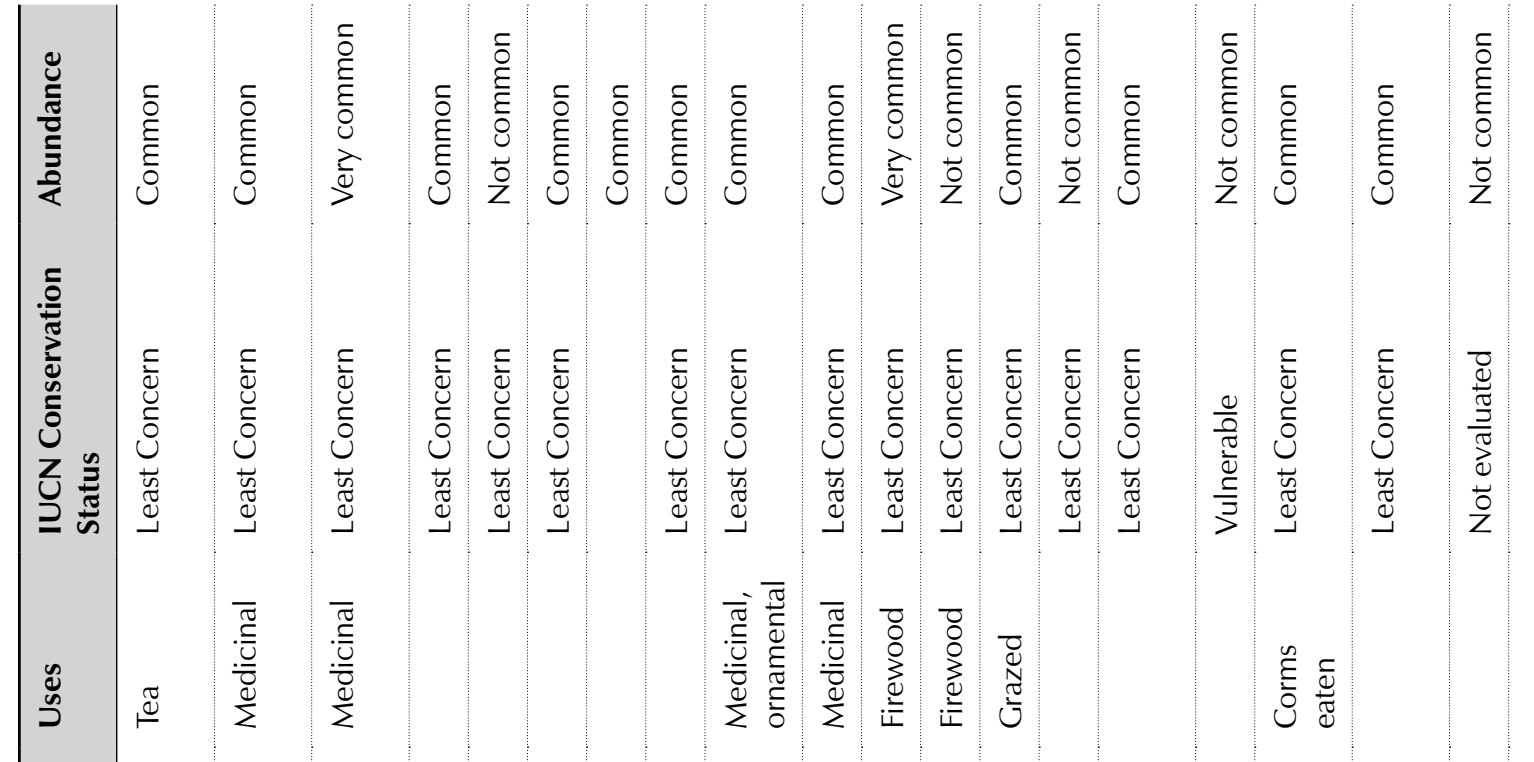

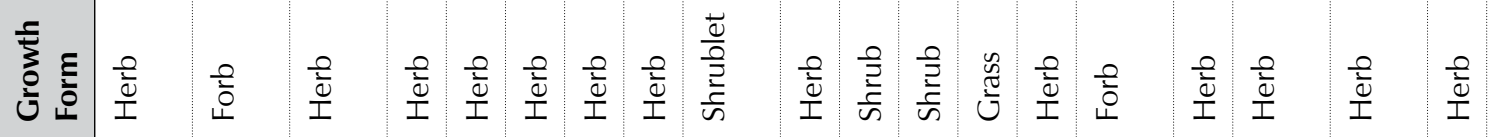

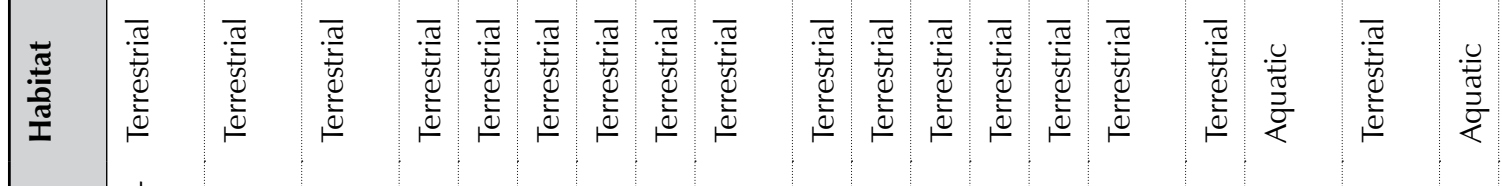

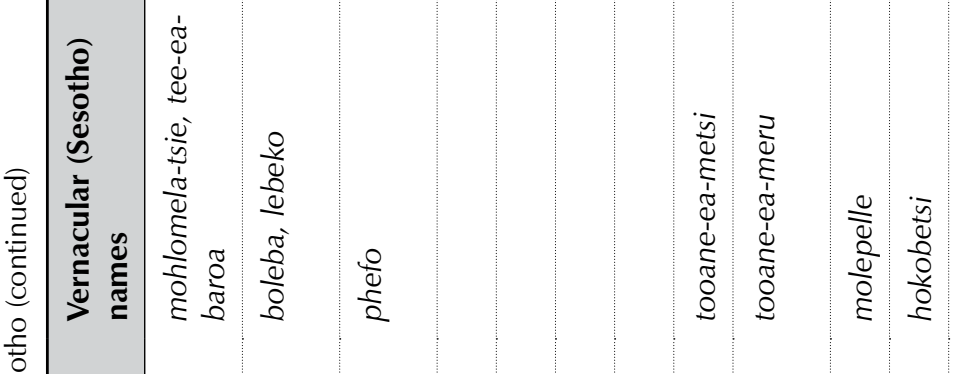

끙

U

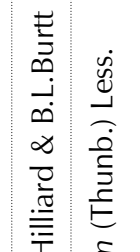

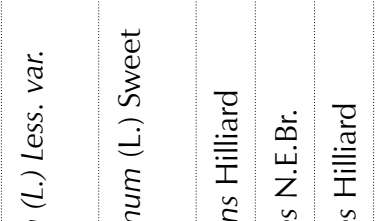

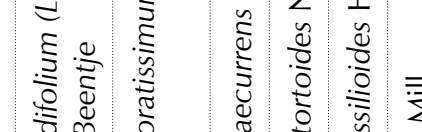

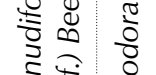

吾

हो $ह$ ह

हो

घ 5

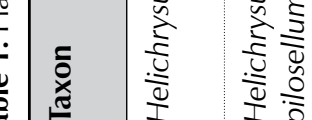

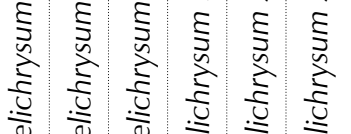

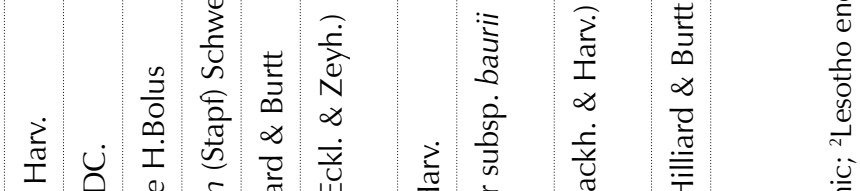

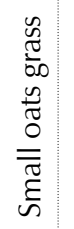

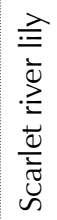

$\frac{\frac{\sqrt{2}}{\frac{\pi}{2}}}{\frac{\pi}{2}}$

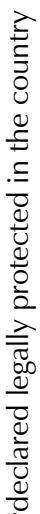

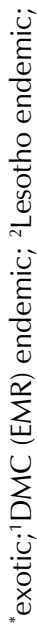




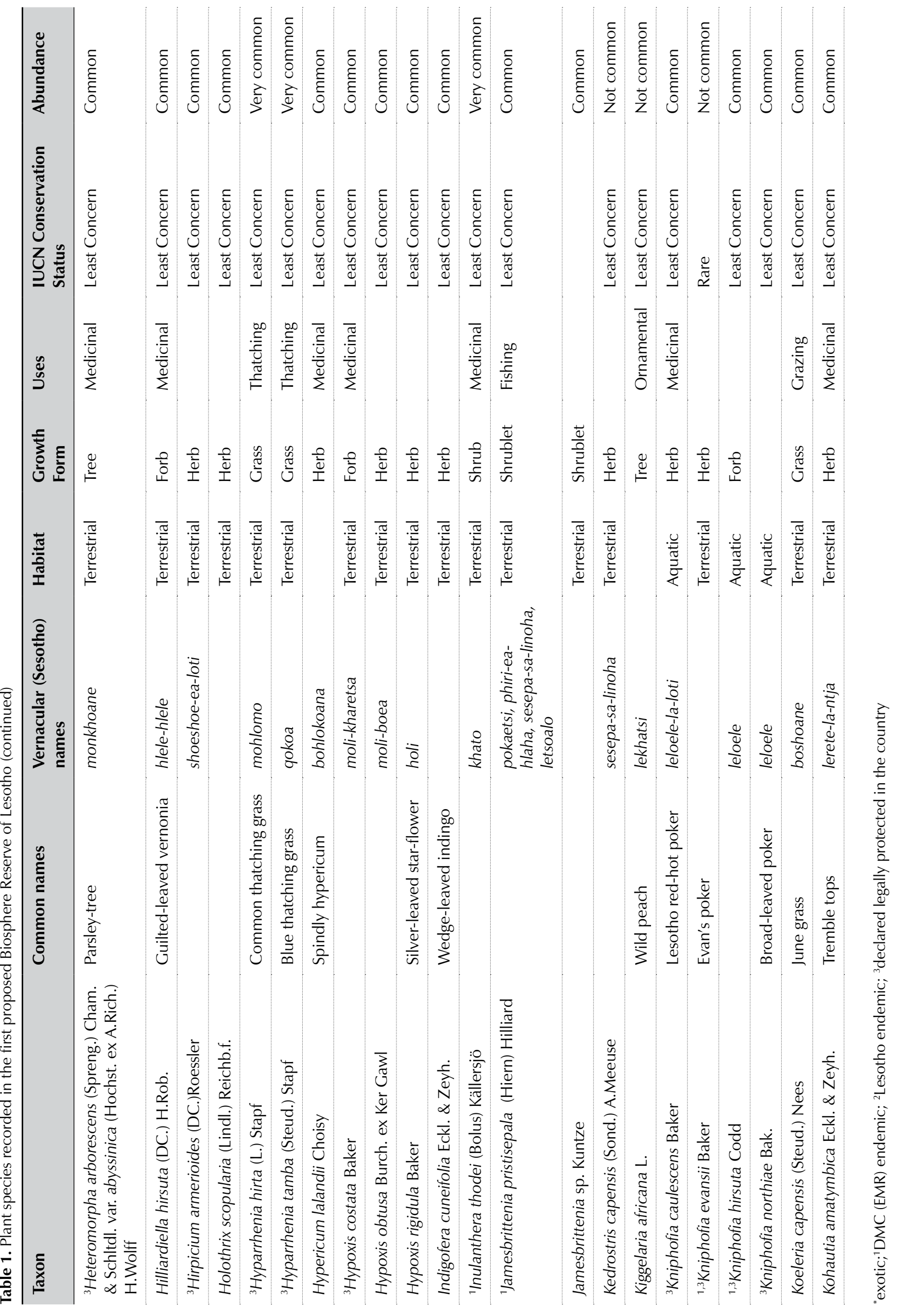




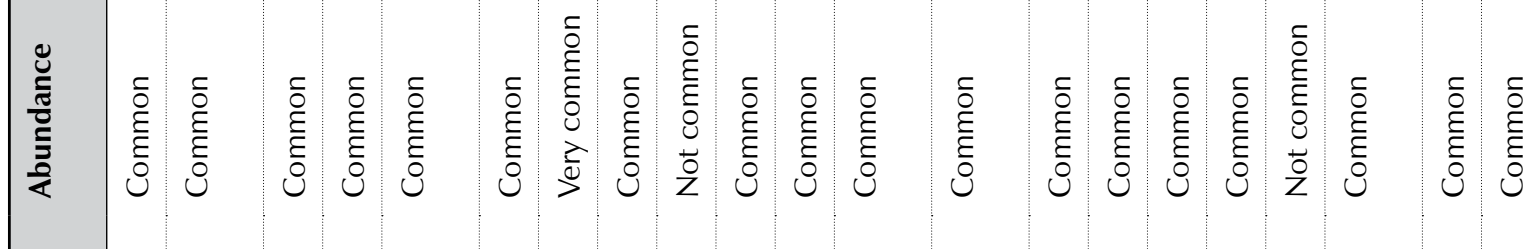

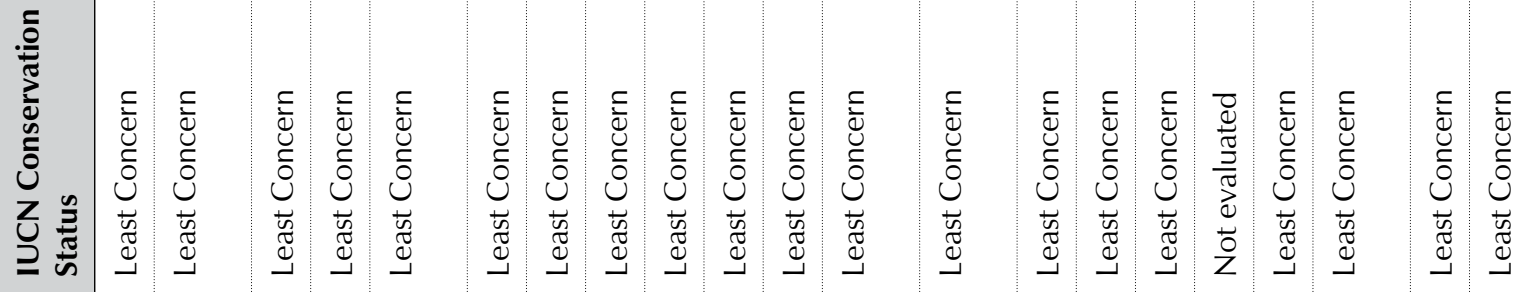

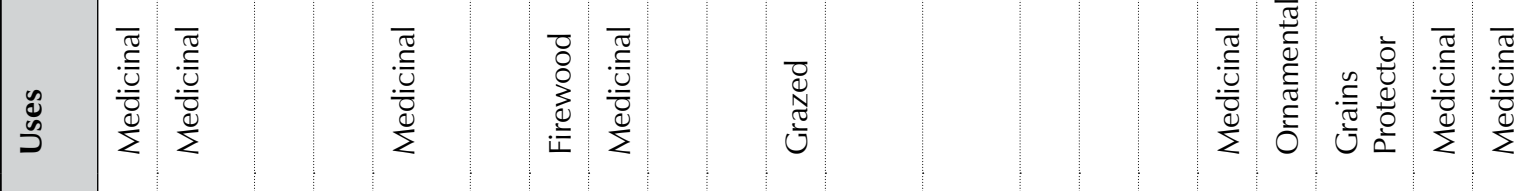

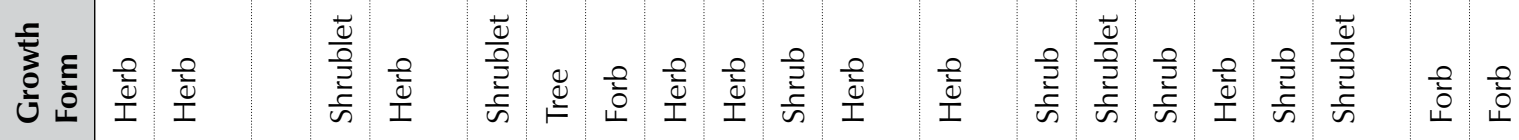

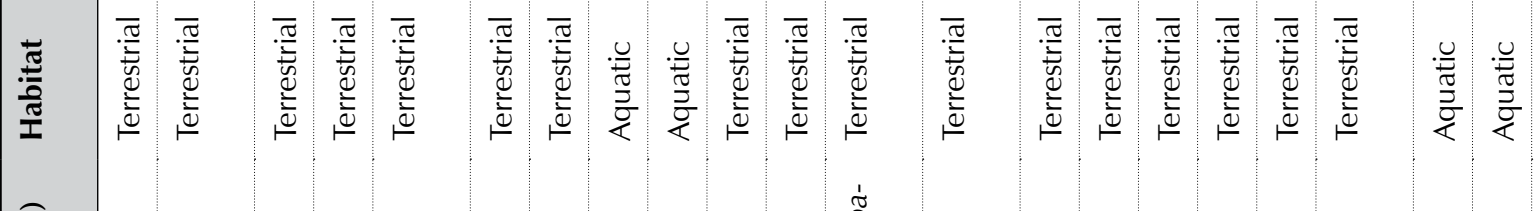

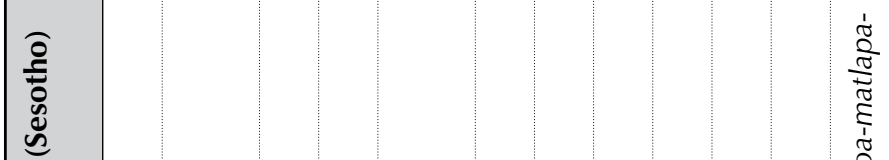

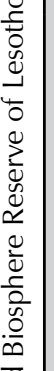

竎

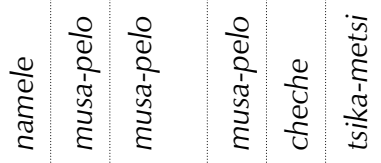

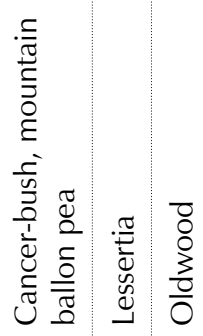

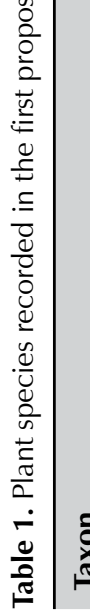

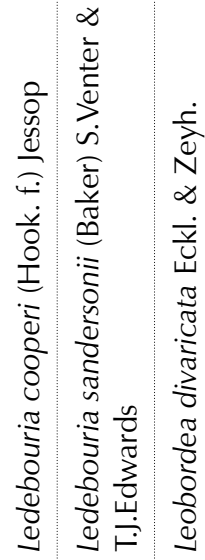

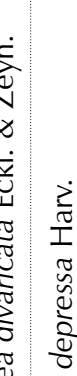

$\underset{⿱ 亠 ⿻}{\infty}$

ن.

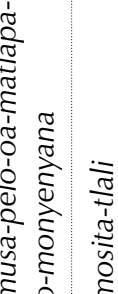

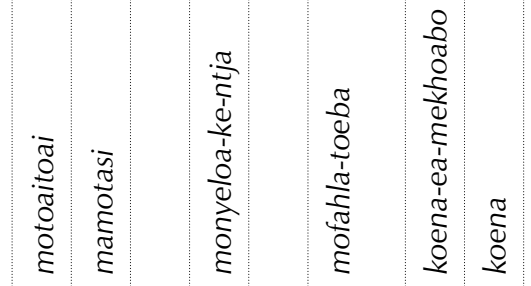

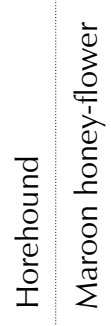

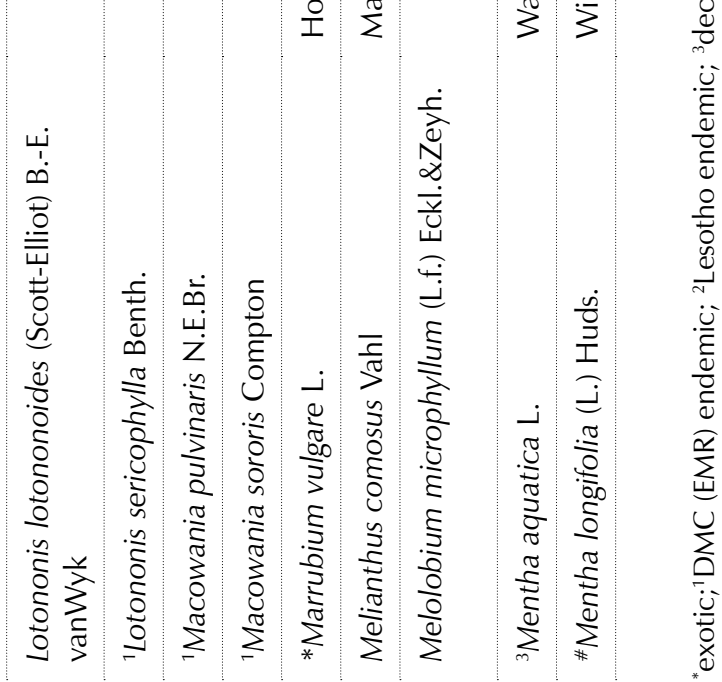

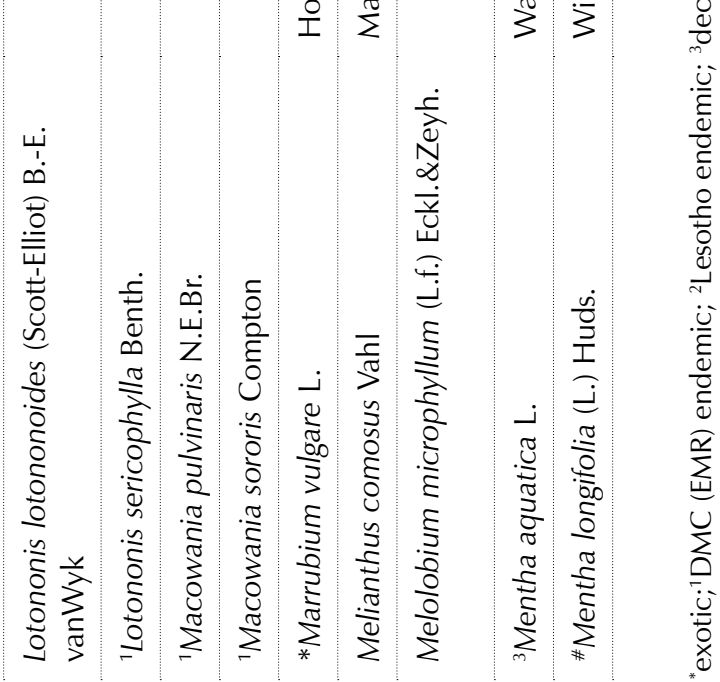

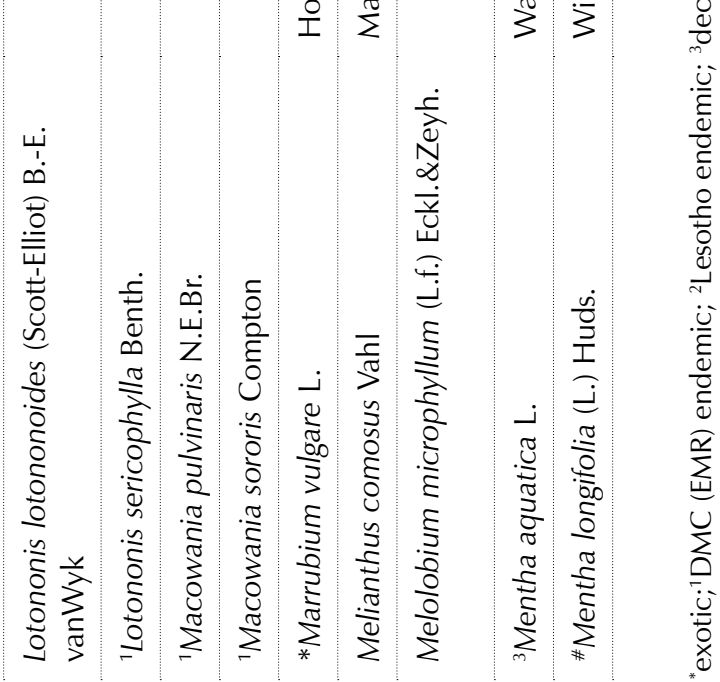

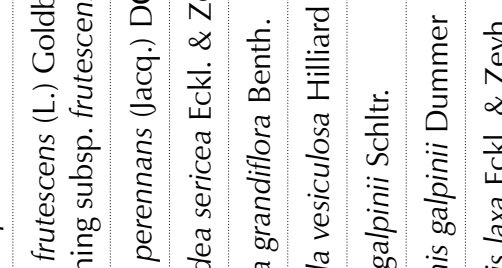

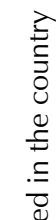




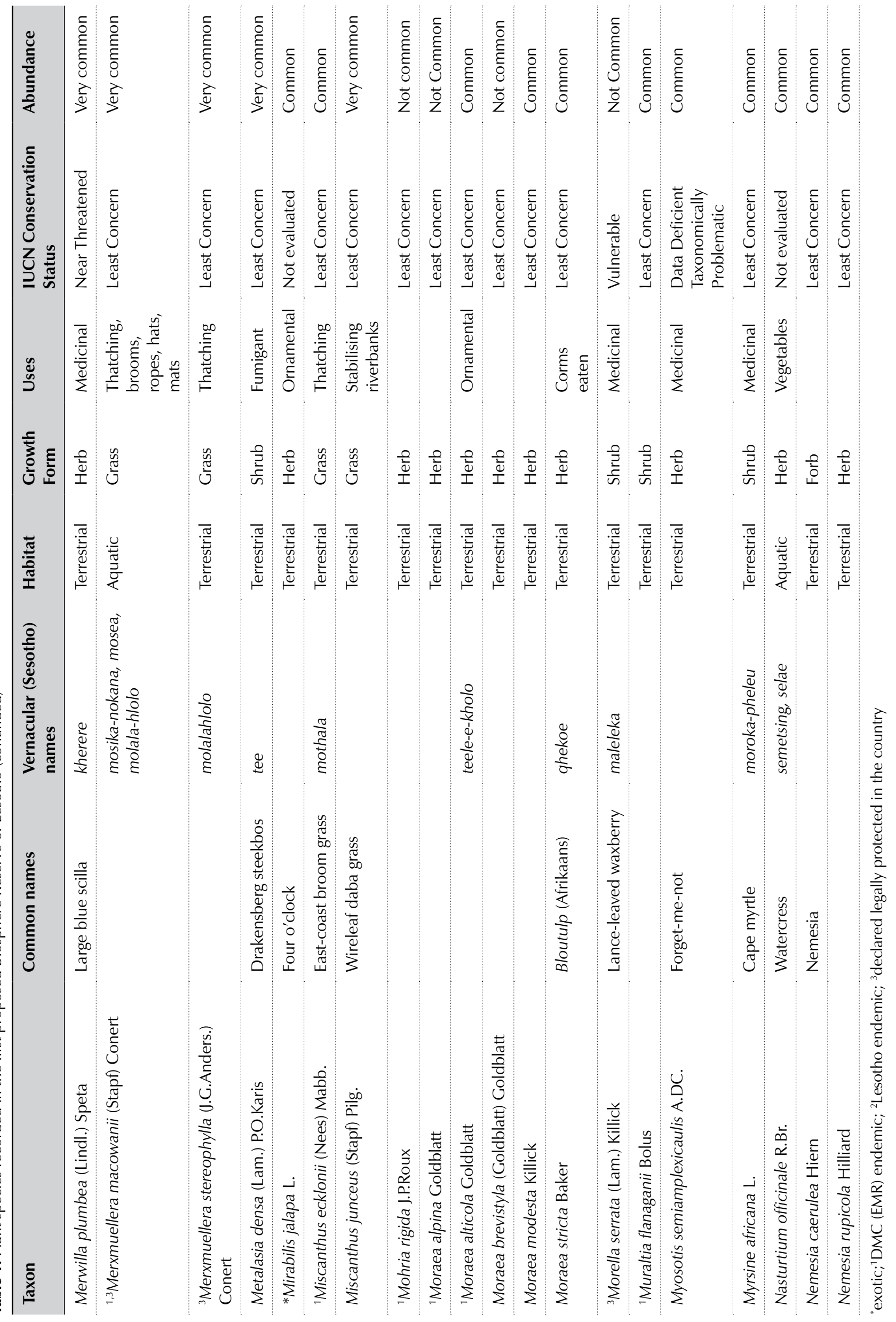




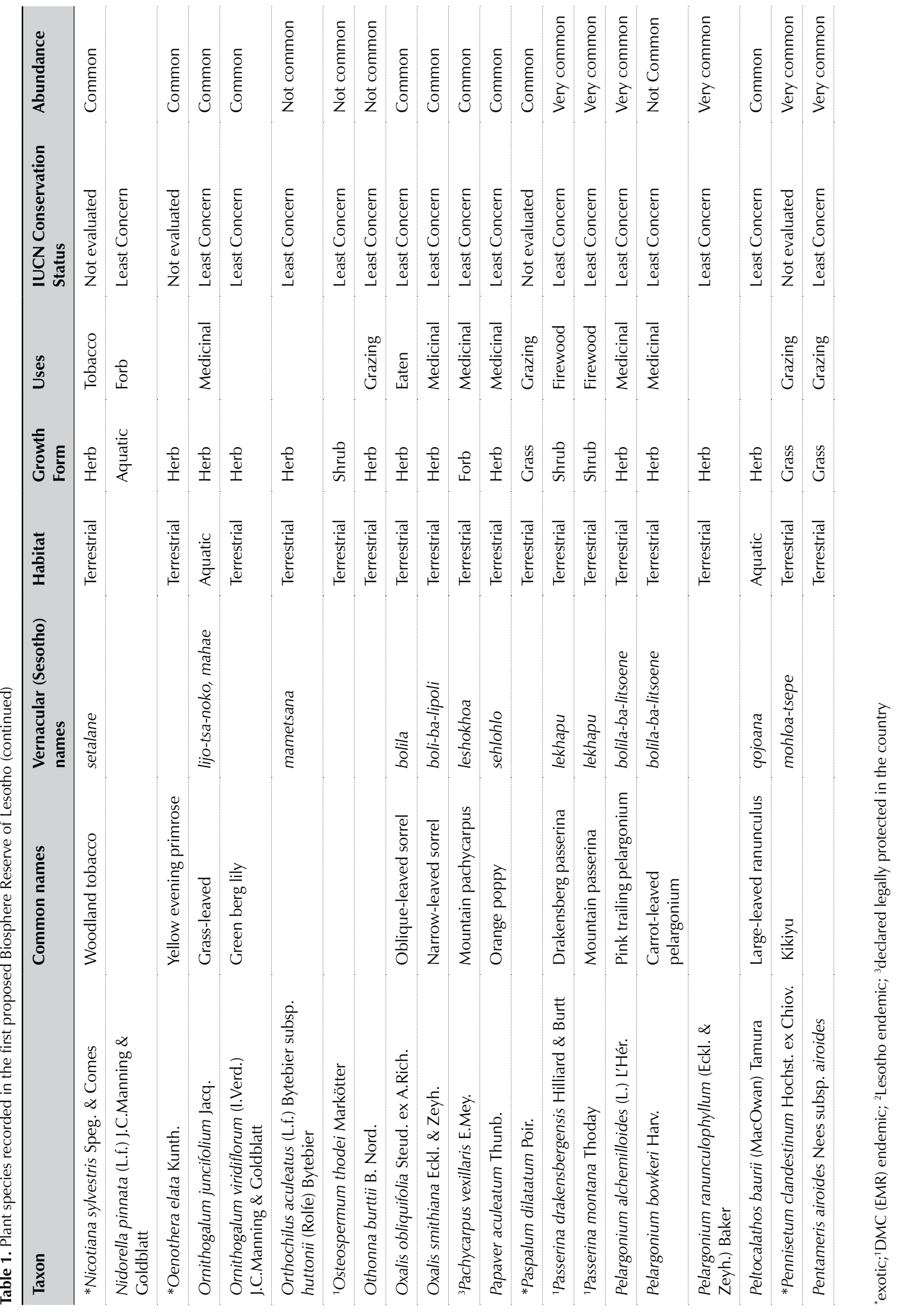




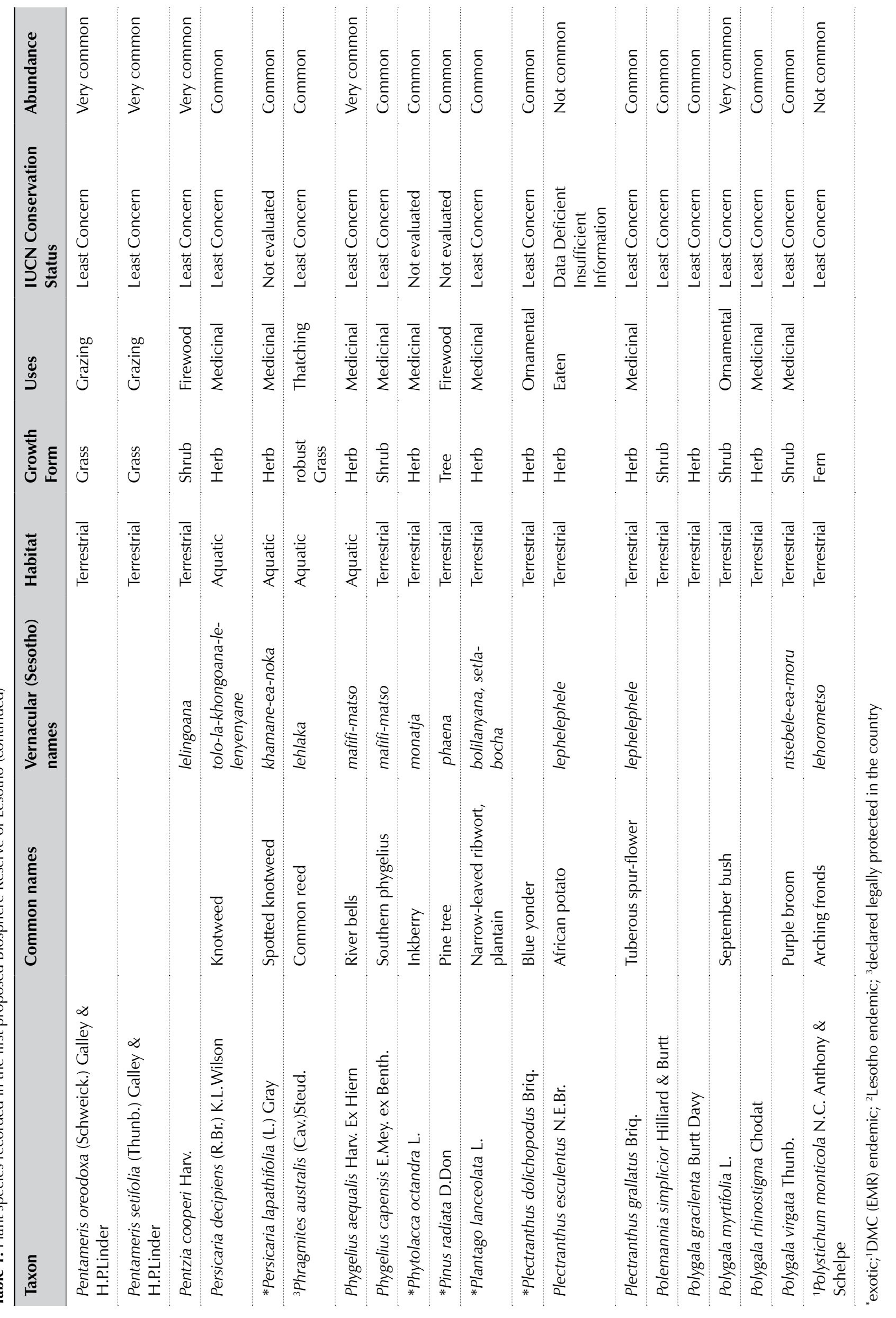




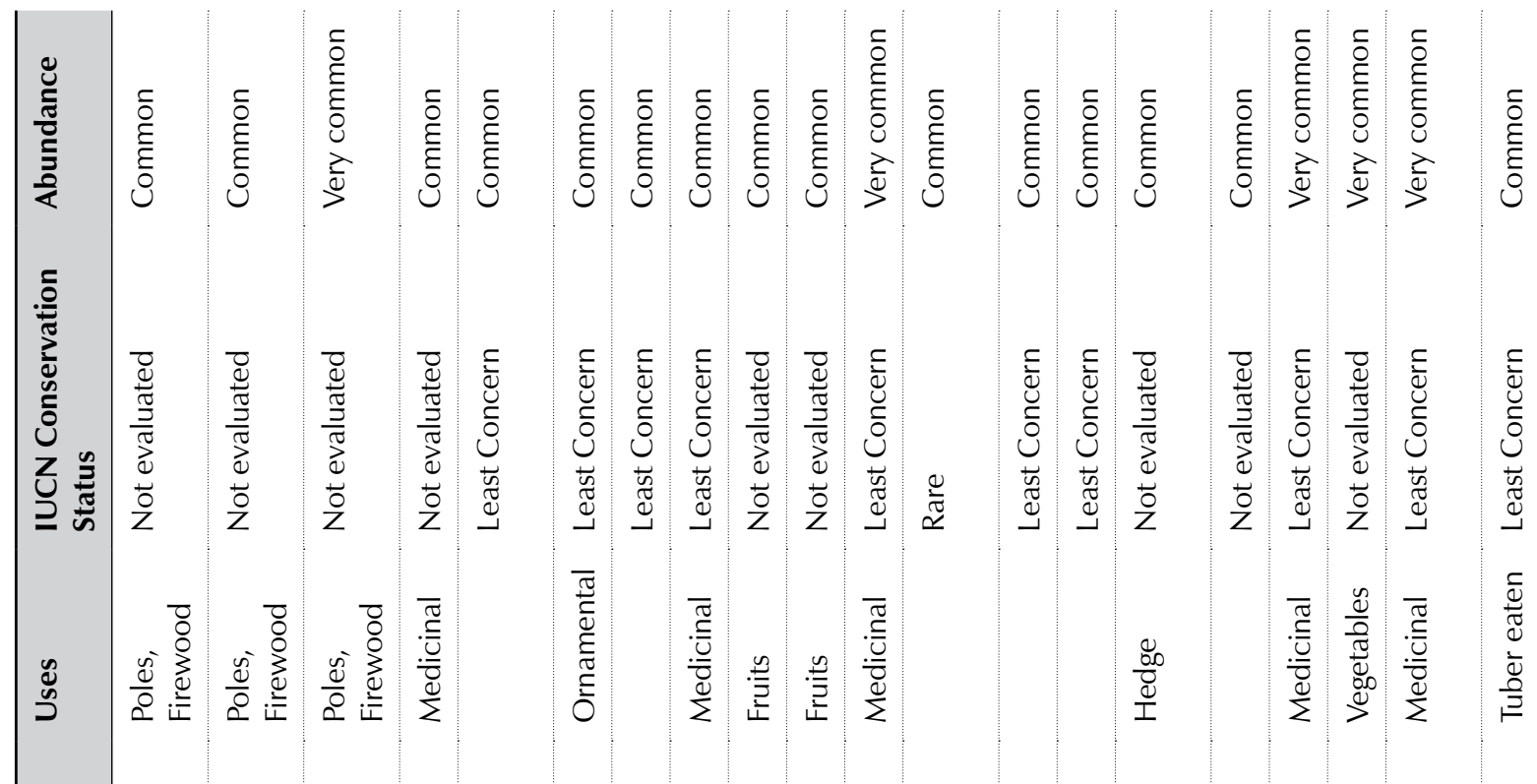

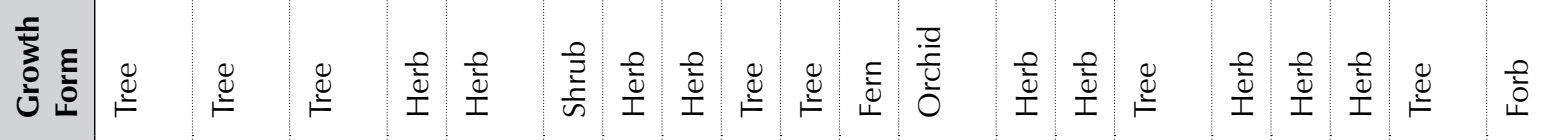

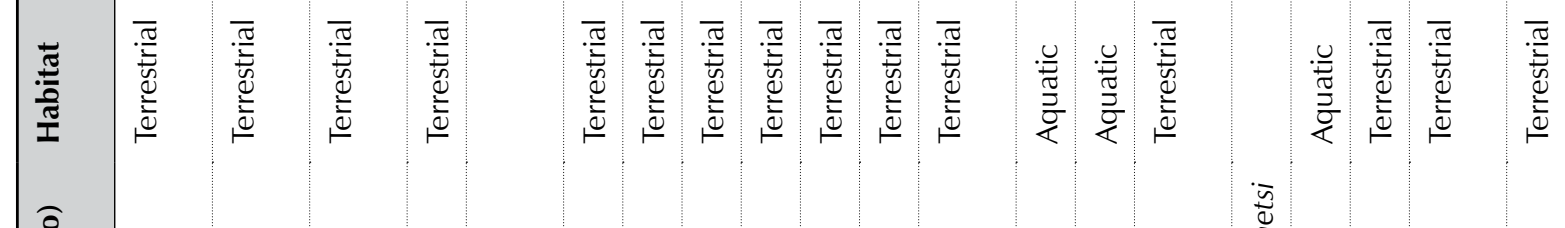

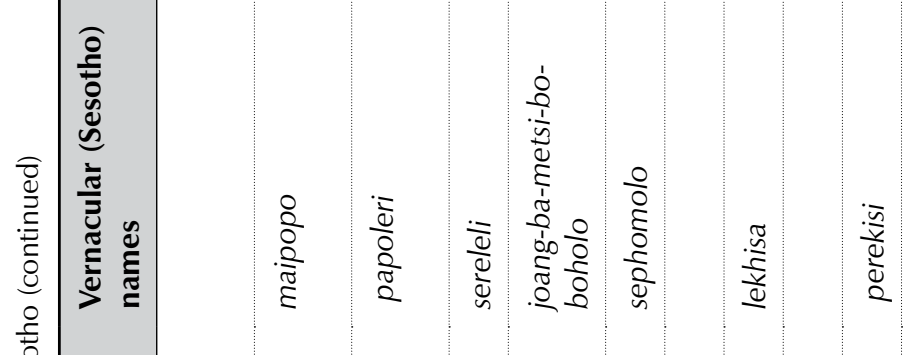

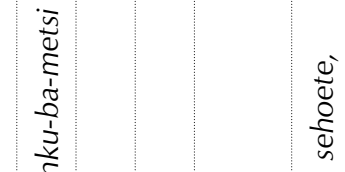

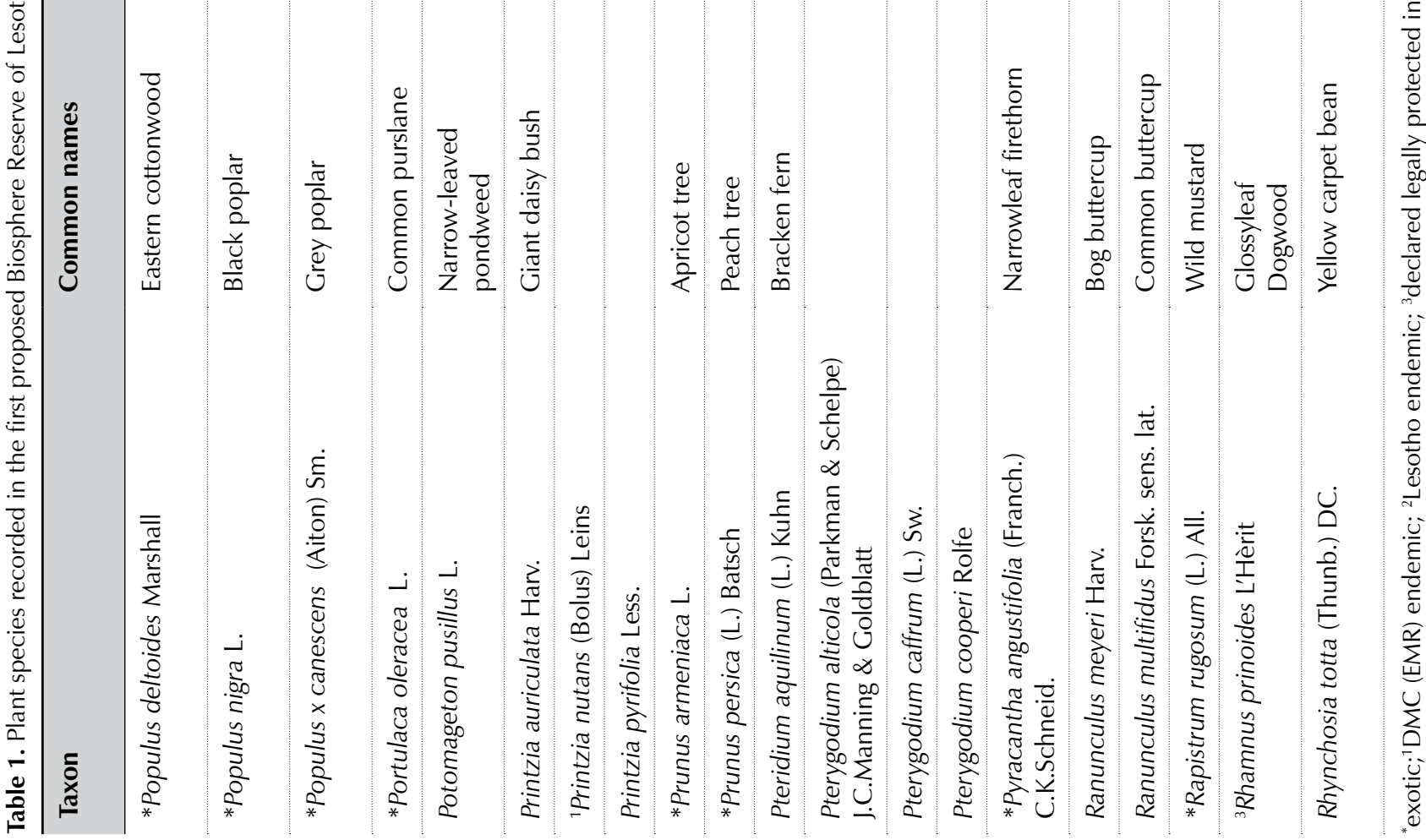




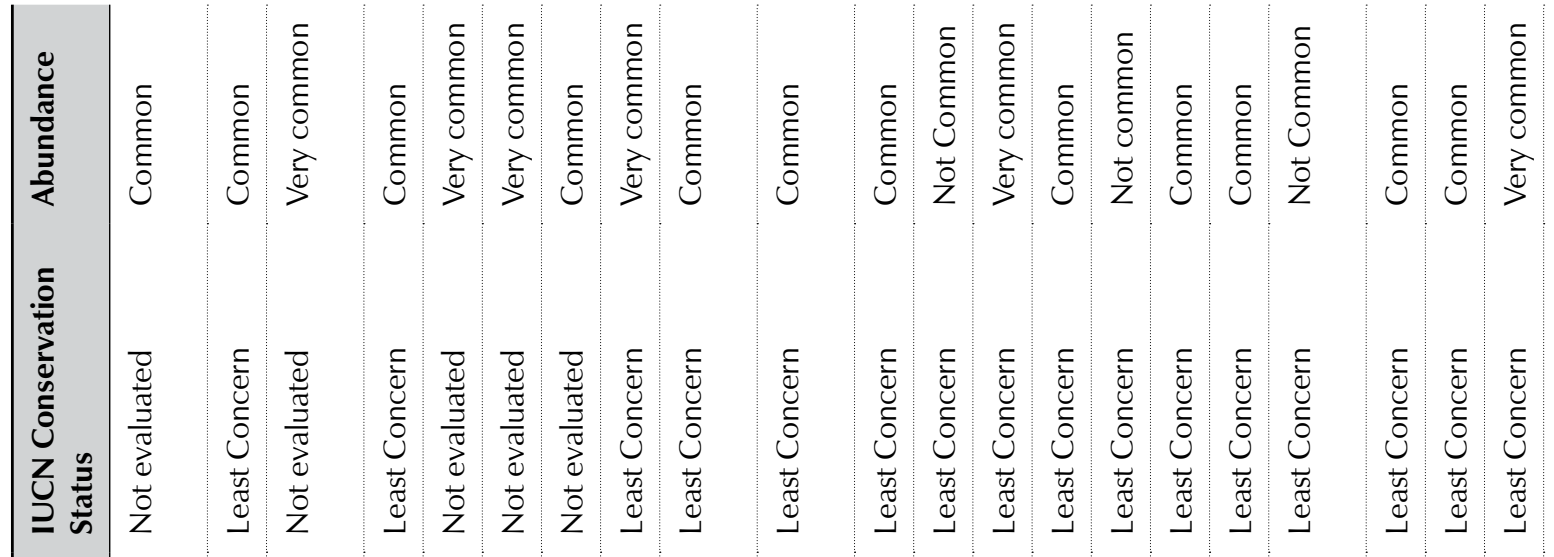

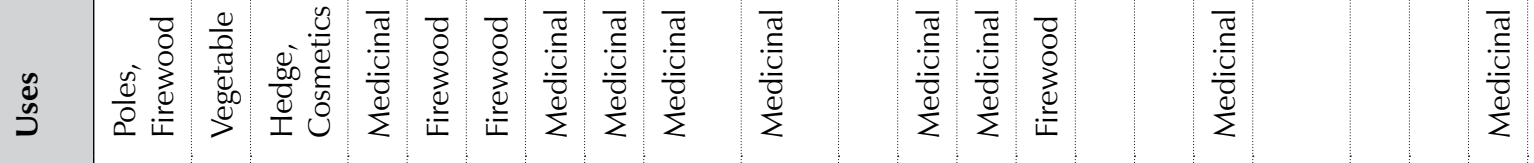

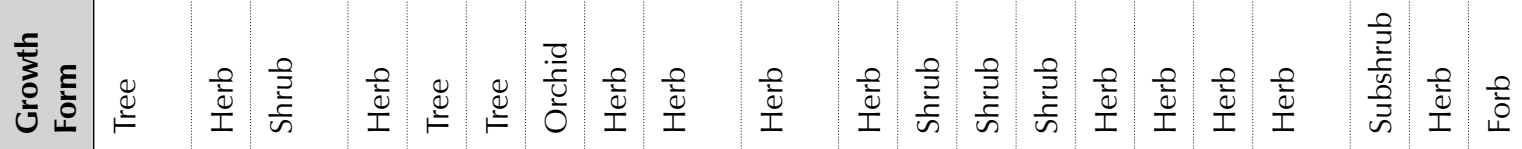

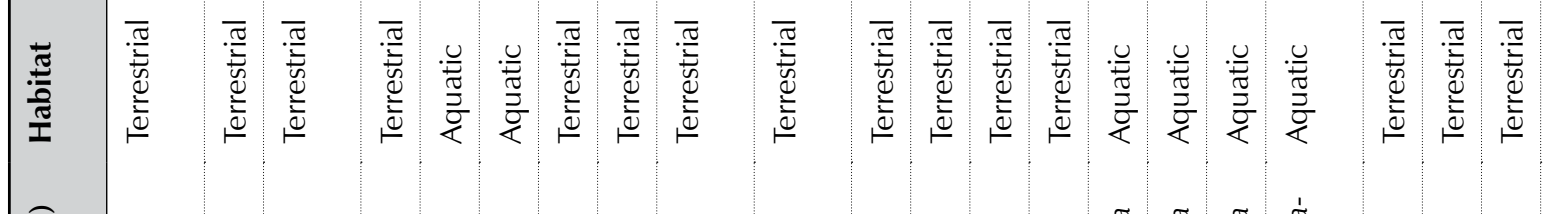

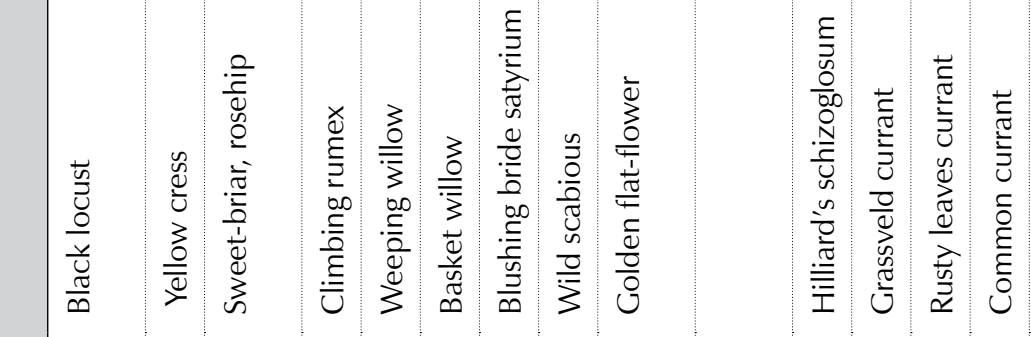

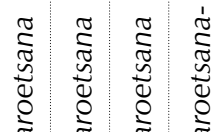

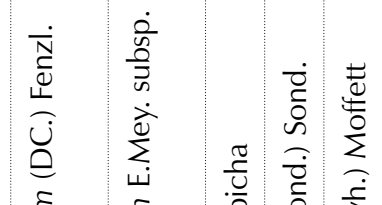




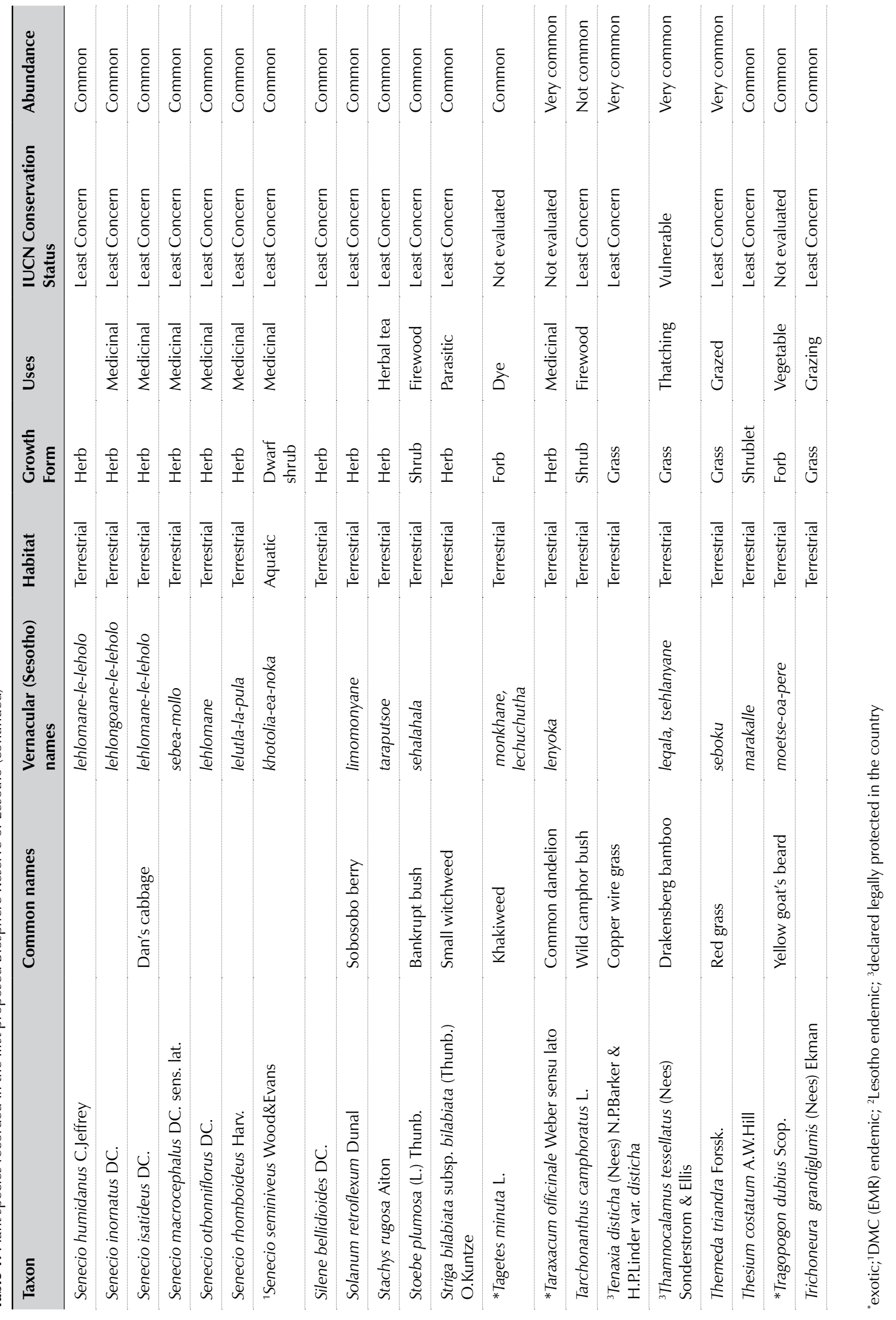




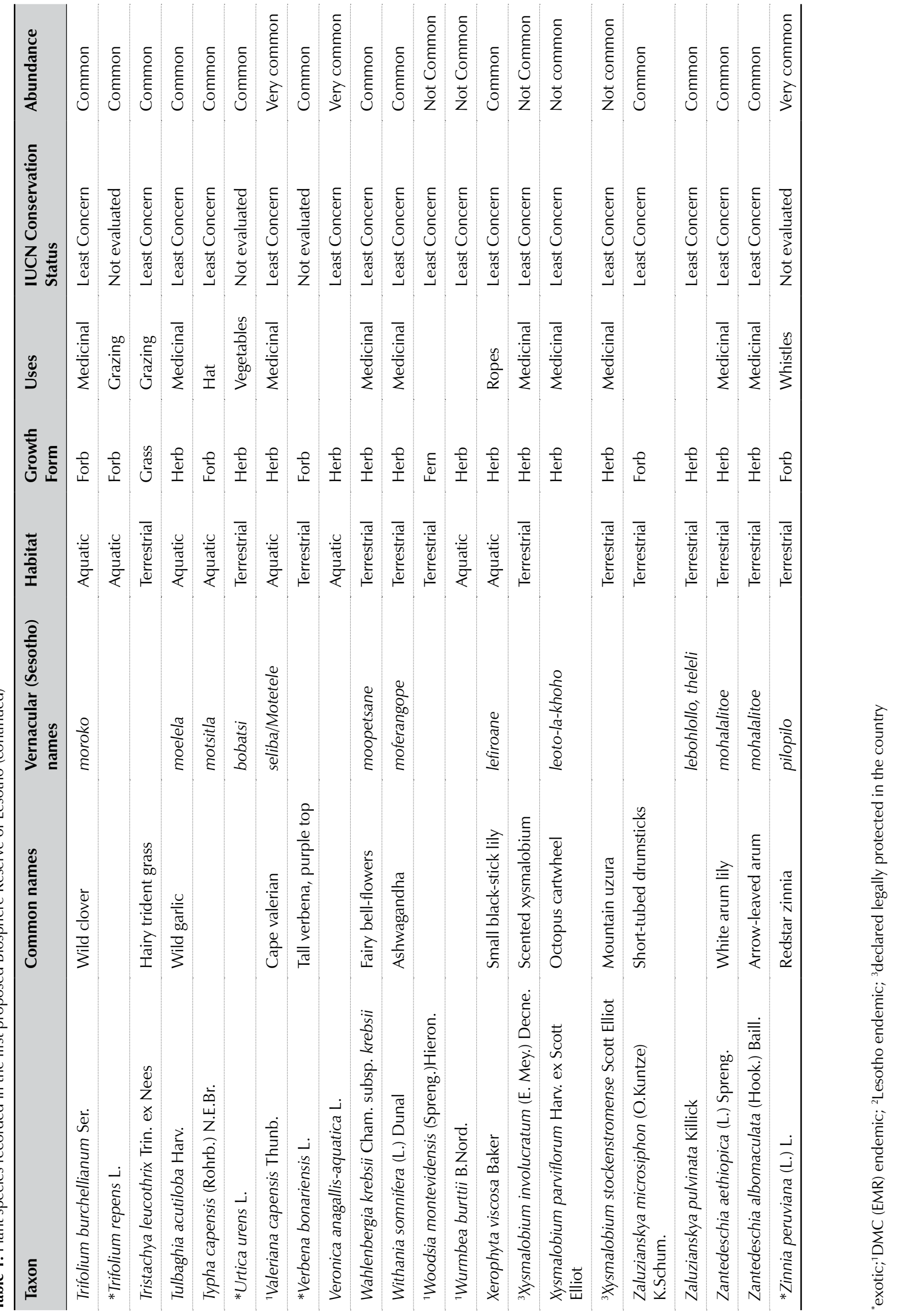


rosehip (Rosa rubiginosa L.). Even though some of the exotic species are invasive, such as yellowcress (Rorippa nudiuscula Thell.) and khakiweed (Tagetes minuta L.), some of these exotic species are of commercial importance. For example, rosehip is consumed for its edible fruits and is also used for medicinal purposes. In fact, Lesotho is currently exporting rosehip fruits to Germany for making tea and jam, as well as for the production of essential oils used in the cosmetics industry. The residue from the plant is reported to induce fertility in animals (Kobisi et al. 2019). Sixty of the 380 recorded species are endemic to the DMC. Two of the recorded species are endemic to Lesotho namely Glumicalyx lesuticus Hillard \& Burtt and spiral aloe (Aloe polyphylla Schönl. ex Pillans, Figure 3C), whereas Othonna burttii B.Nord. and Lesotho lily (Gladiolus saundersii Hook.f., Figure 3D) are near endemic. Spiral aloe is the national flower of Lesotho and is under threat due to illegal trade. Some natural populations are now extinct from certain areas in the country where the plant used to be abundant (pers. obs.). However, it is surprising that spiral aloe is listed as Vulnerable, whereas G. lesuticus and O. burttii have an IUCN conservation status of Least Concern, despite their limited distribution and population in the country. On the other hand, G. lesuticus and O. burttii have not previously been recorded by authors who documented the flora of Lesotho (e.g. Phillips 1917; Jacot Guillarmod 1971; Schmitz 1982; Kobisi 2005), even though these plants have been listed as Lesotho endemics (NES 2000) and are Red Listed (Talukdar 2002).

A notably conspicuous but locally uncommon fern, known as bracken (Pteridium aquilinum (L.) Kuhn, Figure $3 \mathrm{~F}$ ), which has a narrow distribution in Lesotho, was also recorded in TNP. Cooper-Driver et al. (2008) stated that even though bracken is a common weedy plant worldwide, it is surprisingly rare in Lesotho. Bracken was found forming a colony within a thicket of oldwood in TNP at an elevation of 2037 masl, at GPS coordinates of S $28^{\circ} 55^{\prime} 28.5^{\prime \prime}, \mathrm{E} 028^{\circ} 26^{\prime} 44.8^{\prime \prime}$. The only other records of this plant in the country are $\mathrm{Ha}$ Ntsi, on the way to Mohale Dam, and Ha Mamokoaqo in the Leribe District, collected by Dieterlen in 1903 (Cooper-Driver et al. 2008). The fern is unique in that it is very large compared to other common fern species occurring in the country, growing to a height of $1.5 \mathrm{~m}$.

\section{Fauna}

Even though a majority of wild species of fauna has been hunted down close to extinction in different parts of the country, a number of species still exist in the proposed $\mathrm{BR}$, particularly birds and mammals. As a result, many of these species are declared legally protected in the country, and these include antelope, baboons, monkeys, bearded vultures, other birds of prey, cranes, storks and herons. A number of species have been recorded in the current study for the five classes of vertebrates (mammals, birds, fish, reptiles and amphibians), and these are discussed in more detail in the sections that follow.

Mammals: A total of 16 mammal species were recorded in the proposed BR, including rodents, jackal, antelope, dassies, rabbits and baboons (Table 2). Seven of the 16 recorded species are declared legally protected, and these include African clawless otter (Aonyx capensis Schinz, 1821), Cape porcupine (Hystrix africaeaustralis Peters, 1852), chacma baboon (Papio ursinus ursinus (Kerr, 1792)), grey rhebok (Pelea capreolus (Forster, 1790)) and common eland (Tragelaphus oryx (Pallas, 1766)). The common eland seems to have been previously widespread in the country, as evidenced by the many occurrences of rockart (Figures $3 \mathrm{H}, \mathrm{I}$ ). However, the species was locally extirpated mainly due to hunting prior to being reintroduced into TNP following declaration of the area as a PA. In comparison, LHDA (1998a, 1998b) reported 11 mammals in BNR and 12 in TNP, based on reports from field staff in the area. Even though a limited number of mammals have been recorded in the current study, archaeological records indicate that several species of mammals were historically (during the Holocene) widespread in the country (Grab \& Nash 2020). These include aardwolf (Proteles cristata Sparrman, 1783), African wild cat (Felis lybica Forster, 1780), black-backed jackal (Canis mesomelas Schreber, 1775), black wildebeest (Connochaetes gnou (Zimmermann, 1780)), blesbok (Damaliscus pygargus phillipsi Harper, 1939), Cape fox (Vulpes chama (A.Smith, 1833)), common genet (Genetta genetta (Linnaeus, 1758)), scrub hare (Lepus saxatilis F.Cuvier, 1823), klipspringer (Oreotragus oreotragus (Zimmermann, 1783)), mountain reedbuck (Redunca fulvorufula (Afzelius, 1815)), red hartebeest (Alcelaphus buselaphus (Pallas, 1766)), rock hyrax (Procavia capensis (Pallas, 1766)), slender mongoose (Galerella sanguinea (Rüppell, 1835)), springbok (Antidorcas marsupialis (Zimmermann, 1780)), Cape porcupine (H. africaeaustralis), steenbok (Raphicerus campestris (Thunberg, 1811)) and common warthog (Phacochoerus africanus (Gmelin, 1788)) (Grab \& Nash 2020). Given the drastic extinction rate of fauna species in Lesotho, the recording of six of these species in the current study, namely African wild cat, black-backed jackal, common genet, scrub hare, rock hyrax and Cape porcupine, adds to the biodiversity value of the proposed BR. However, absence of some of these species currently reveals a major loss of fauna in Lesotho over the past 200 years as discussed by Grab and Nash (2020).

Birds: Fifty-three bird species were recorded in the proposed BR, 13 of which are declared legally protected in the country (Table 3). It is important to note that two of the 13 protected species, namely Cape Vulture (Gyps coprotheres (Forster, 1798)) and Bearded Vulture (Gypaetus barbatus (Linnaeus, 1758)), have a limited distribution and are globally listed as Endangered and Near Threatened respectively (Hockey et al. 2006). Indeed LHDA (1998a, 1998b) reported a total of ten 
Table 2. Mammals species recorded in the first proposed Biosphere Reserve of Lesotho

\begin{tabular}{|c|c|c|c|c|c|}
\hline Taxon & Common names & $\begin{array}{l}\text { Vernacular } \\
\text { (Sesotho) names }\end{array}$ & $\begin{array}{l}\text { IUCN } \\
\text { Conservation } \\
\text { Status }\end{array}$ & Habitat & Abundance \\
\hline${ }^{3}$ Aonyx capensis Schinz & African clawless otter & qibi & $\begin{array}{l}\text { Near } \\
\text { Threatened }\end{array}$ & Aquatic & Not Common \\
\hline Canis mesomelas Schreber & Black-backed jackal & Phokojoe & Least Concern & Terrestrial & Not Common \\
\hline${ }^{3}$ Caracal caracal Schreber & Caracal & Thoalere & Least Concern & Terrestrial & Not Common \\
\hline $\begin{array}{l}\text { Cryptomys hottentotus } \\
\text { Lesson }\end{array}$ & $\begin{array}{l}\text { Common african } \\
\text { mole-rat }\end{array}$ & Mokunyane & Least Concern & Terrestrial & Common \\
\hline Felis lybica Schreber & African wild cat & & Least Concern & Terrestrial & Not Common \\
\hline $\begin{array}{l}{ }^{3} \text { Galerella pulverulenta } \\
\text { Wagner }\end{array}$ & Small grey mongoose & Mochalla & Least Concern & Terrestrial & Common \\
\hline Genetta genetta L. & Small-spotted genet & Qoako & Least Concern & Terrestrial & Not Common \\
\hline${ }^{3}$ Hystrix africaeaustralis Peters & Cape porcupine & Noko & Least Concern & Terrestrial & Not Common \\
\hline Ictonyx striatus Perry & Striped polecat & Nakeli & Least Concern & Terrestrial & Not Common \\
\hline Leptailurus serval Schreber & Serval & & Least Concern & Terrestrial & Not Common \\
\hline Lepus saxatilis F. Cuvier & Scrub hare & Mutlanyana & Least Concern & Terrestrial & Common \\
\hline $\begin{array}{l}\text { Mystromys albicaudatus } \\
\text { Smith }\end{array}$ & White-tailed rat & & Vulnerable & Terrestrial & Not Common \\
\hline Otomys sloggetti Thomas & Sloggett's ice rat & Leboli-leqhoa & Least Concern & Terrestrial & Very Common \\
\hline $\begin{array}{l}{ }^{3} \text { Papio cynocephalus ursinus } \\
\text { Kerr }\end{array}$ & Chacma baboon & Tsoene & Least Concern & Terrestrial & Not Common \\
\hline${ }^{3}$ Pelea capreolus Forster & Grey rhebok & Letsa & $\begin{array}{l}\text { Near } \\
\text { Threatened }\end{array}$ & Terrestrial & Common \\
\hline Procavia capensis Pallas & Rock dassie & Pela & Least Concern & Terrestrial & Very Common \\
\hline Pronolagus rupestris A.Smith & Red rock rabbit & Thlolo & Least Concern & Terrestrial & Common \\
\hline${ }^{3}$ Tragelaphus oryx Pallas & Common eland & Phoofu & Least Concern & Terrestrial & Not Common \\
\hline
\end{tabular}

${ }^{3}$ Declared legally protected in the country

Red-listed bird species, including the Cape Vulture and Bearded Vulture in both BNR and TNP. Even though their common breeding sites are the high elevation cliffs in Lesotho, the two bird species occassionally migrate to neighbouring countries such as South Africa, Botswana, northern Namibia and southern Zimbabwe (Hockey et al. 2006). Lesotho has also legally protected the two bird species (under Legal Notice No. 36 of 1969, as amended by Legal Notice No. 93 of 2004 and No. 38 of 2006), as well as listing them under the Convention on International Trade in Endangered Species of Wild Fauna and Flora (CITES).

Lesotho is reported to be the main breeding ground to the rare Bearded Vulture in southern Africa (NES 2000). A study by Donázar et al. (1993) revealed that elevation and ruggedness of the topography probably influence the existence of adequate breeding places for the Bearded Vulture, as is the case in the proposed $\mathrm{BR}$, however, human disturbance negatively affects the breeding success of the bird. In fact, the population of the species has declined drastically, mainly due to hunting of adults and robbery of eggs and chicks, as well as fire, decline in food supply, gin traps and collection of the birds for skins and plumage. In addition, since the Bearded Vulture is a specialised species in terms of its habitat requirements and food choice, Colahan (2004) suggested establishment of partnerships between conservation organisations for management and conservation of the population. Several efforts are currently being undertaken by the Department of Environment in Lesotho (of the Ministry of Tourism, Environment and Culture) together with South Africa, under a bilateral programme targeted towards enhanced breeding of the species. A Bearded Vulture Management Plan has also been developed for conservation of the species.

The decline of Bearded Vulture populations has also been observed in other parts of the world. For example, the decline is reported to have started in the mid-nineteenth century in Europe and North America, 


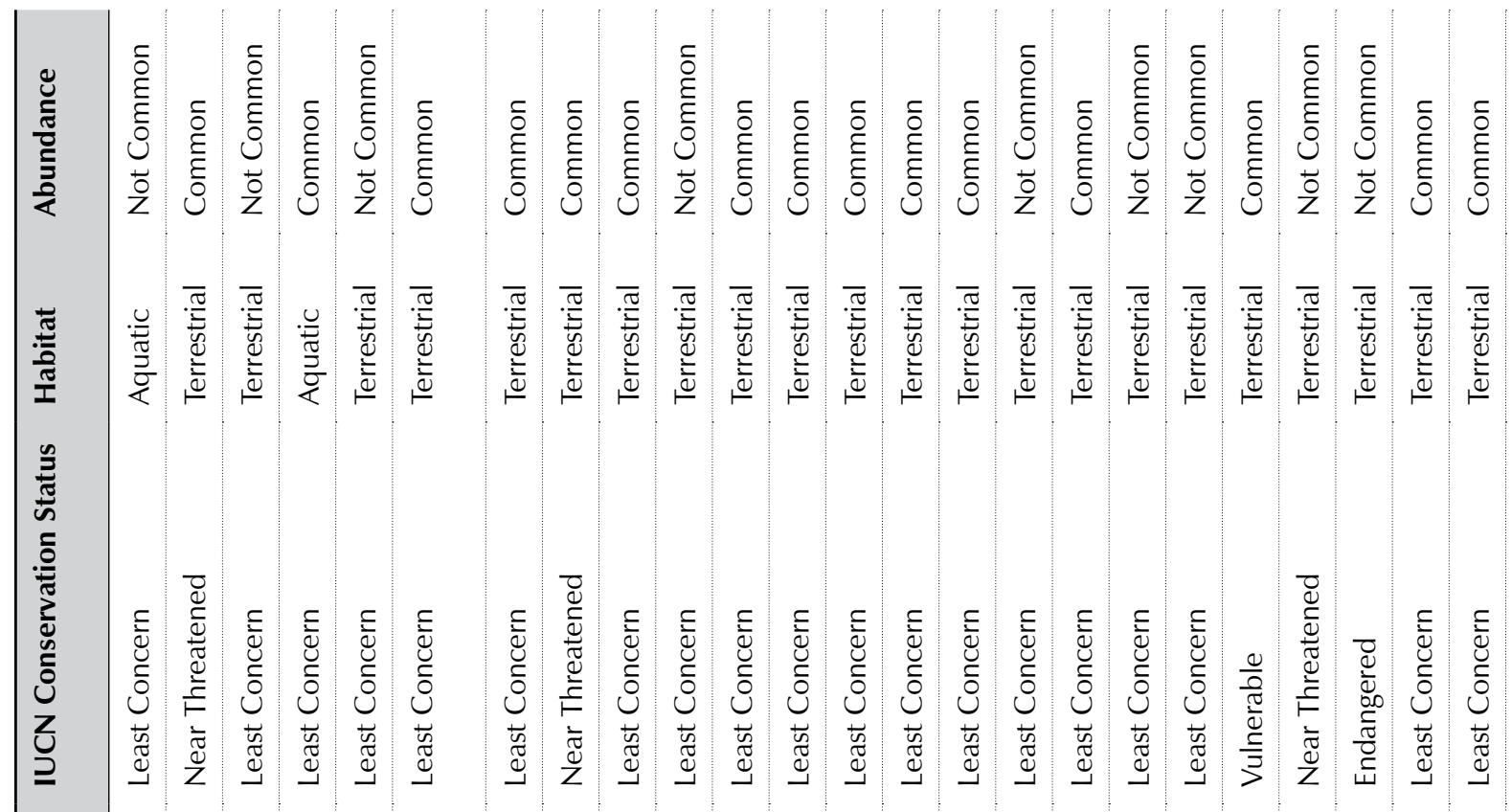

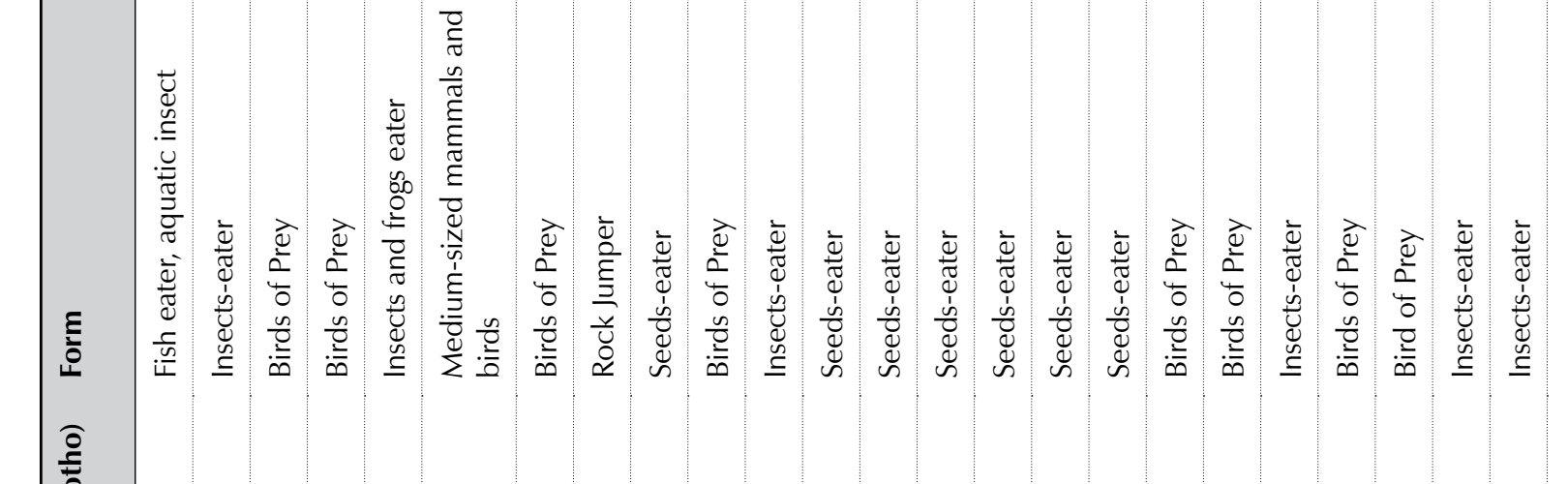




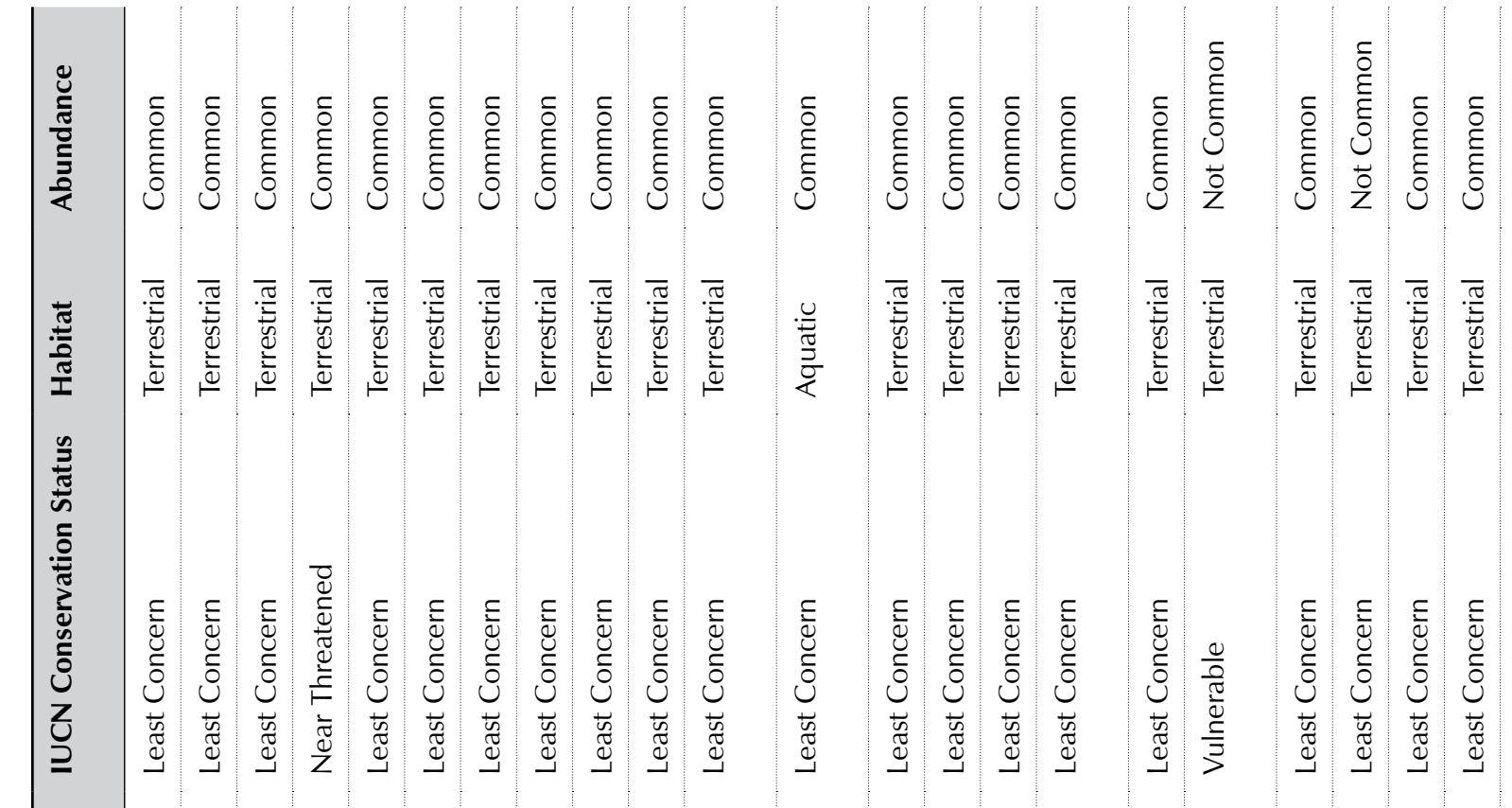

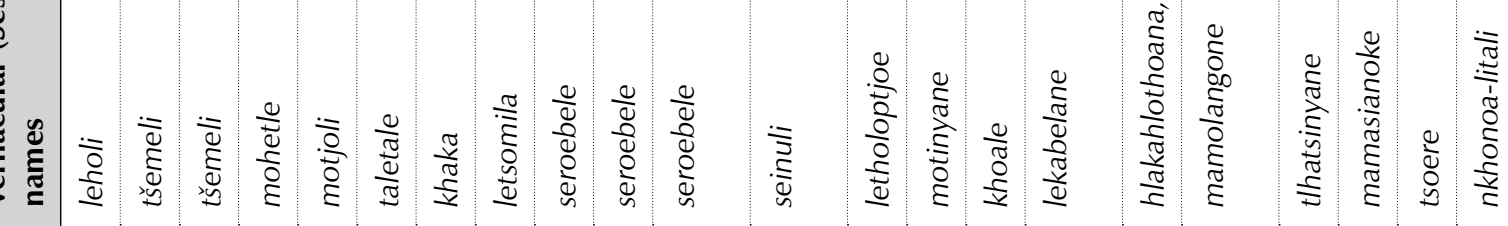

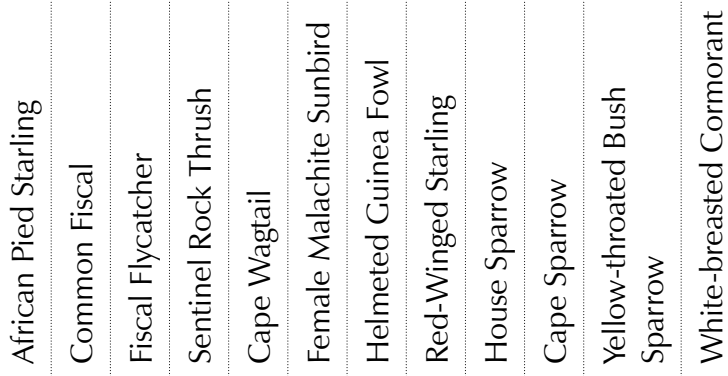

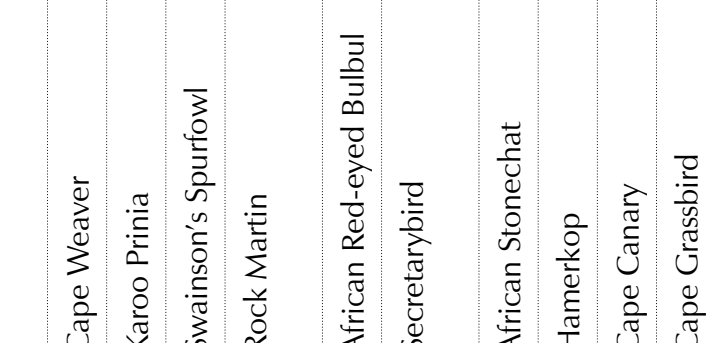

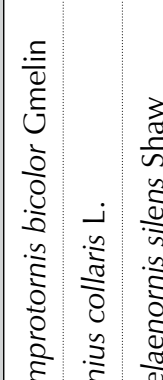

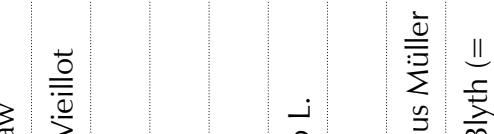
है

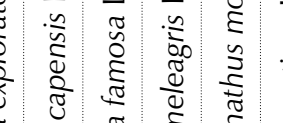

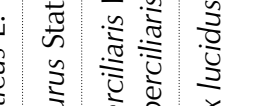

$$
\text { . }
$$

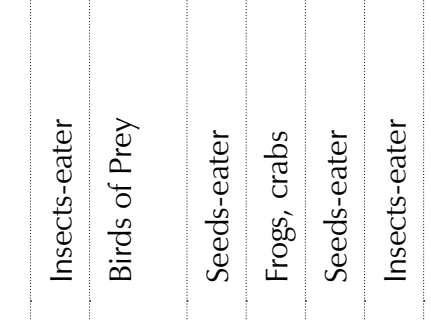




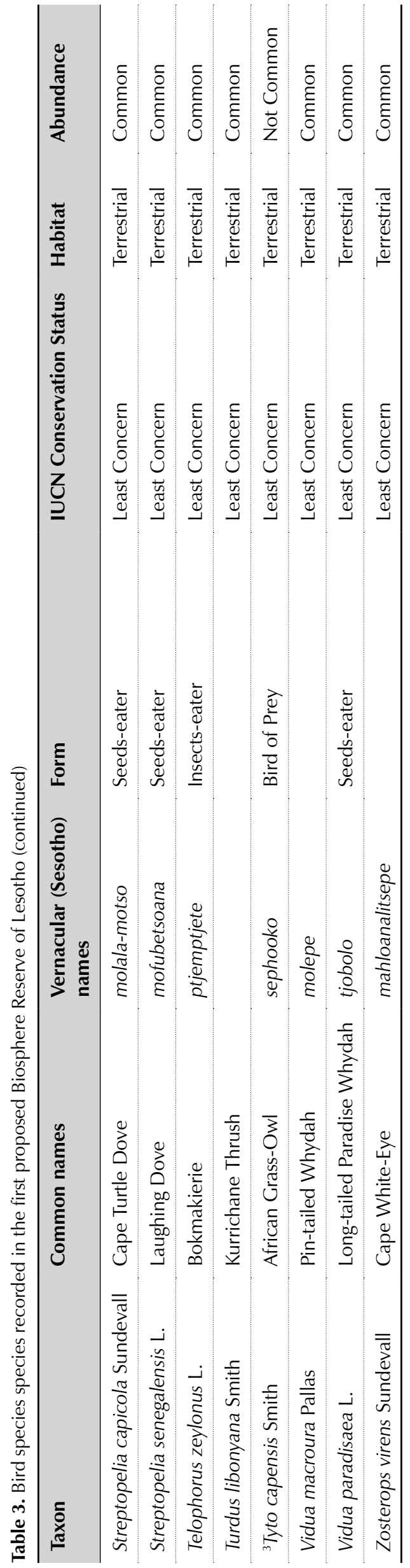

causing the populations to approach extinction (Ogada et al. 2012). A similar observation has also been made in the Middle East, as well as Central and South America (Ogada et al. 2012). According to Di Vittorio et al. (2018), vulture populations in West Africa are also undergoing dramatic decline, particularly due to factors such as environmental changes, poisoning and bioaccumulation of toxic substances from agricultural products, pesticides, and veterinary drugs used in cattle livestock. In addition, the vultures are subject to direct persecution for the trade of products used in traditional medicine (Di Vittorio et al. 2018). The proposed BR would assist in creating a platform for enhanced breeding of the species to increase the population size. In addition, raising awareness of the biodiversity wealth of the area amongst all residents and role-players could potentially result in reduced utilisation of natural resources specifically within the core areas. Interestingly, Birdlife International categorises the Lesotho Highlands (under which the proposed BR falls) as an Endemic Bird Area of high priority and lists the Drakensberg Rockjumper (Chaetops aurantius Layard, 1867), Mountain Pipit (Anthus hoeschi Stresemann, 1938) and Drakensberg Siskin (Crithagra symonsi (Roberts, 1916)) to be of particular global interest (LHDA 1998a, 1998b). Therefore the proposed BR has potential to become a popular spot for birders who are interested in high elevation species, and thus enhance tourism in the area.

Fish: Only two fish species were recorded in the current study namely Maloti minnow or Maloti redfin (Pseudobarbus quathlambae (Barnard, 1938)), (Figure 3G) and rainbow trout (Oncorhyncus mykiss (Walbaum, 1792)), as summarized in Table 4. It is reported that Maloti minnow was first discovered in the headwaters of the uMkhomazana River in Kwazulu-Natal, South Africa (Barnard 1938), but was subsequently feared extinct (Jubb 1971). The fish was rediscovered at Sehlaba-thebe in Lesotho in 1970 (Jubb 1971). However, although the South African population was feared to be certainly extinct, the species was recently rediscovered in the Mzimkhulu River system in KwaZulu-Natal (Kubheka et al. 2017) and therefore considered near endemic in Lesotho. The recording of Maloti minnow in the Bokong River during this survey adds tremendous clout to the biodiversity rationale of the proposed BR, particularly because the species is Lesotho's only known endemic vertebrate. However, rainbow trout have been introduced in the country. Indeed, LHDA (1998b) indicated that there was circumstantial evidence that rainbow trout were previously introduced into the Tšehlanyane River, and that a residual population existed downstream of TNP. Therefore, the occurrence of the two fish species in two separate rivers, minimises the possibility of one species being a threat to the other (feeding on the other).

Reptiles: A total of seven reptiles were recorded in the proposed BR, comprising four snakes and three lizards (Table 5). Some of the snakes are venomous, namely 
Table 4. Fish species recorded in the first proposed Biosphere Reserve of Lesotho

\begin{tabular}{lllll}
\hline Taxon & Common names & $\begin{array}{l}\text { Vernacular } \\
\text { (Sesotho) names }\end{array}$ & $\begin{array}{l}\text { IUCN Conservation } \\
\text { Status }\end{array}$ & Abandance \\
\hline${ }^{*}$ Oncorhynchus mykiss Walbaum & Rainbow trout & trautu & Not Evaluated & common \\
\hline${ }^{2}$ Pseudobarbus quathlambae Barnard & Maloti minnow & thoboshana & Endangered & common \\
\hline
\end{tabular}

*Introduced species; ${ }^{2}$ Lesotho endemic

berg adder (Bitis atropos (Linnaeus, 1758)), puff adder (Bitis arietans (Merrem, 1820)) and rinkhals (Hemachatus haemachatus (Bonnaterre, 1790)). Berg adder is known from high elevations in the Maloti-Drakensberg, but also occurs at lower elevations in the Cape Fold Mountains of the Western Cape of South Africa (Barlow et al. 2019). On the other hand, LHDA (1998a, 1998b) previously reported six lizards and four snakes in both BNR and TNP, including the Essex's mountain lizard (Tropidosaura essexi Hewitt, 1927), which is known only from the summit slopes of the KwaZulu-Natal Drakensberg, the adjacent Free State province and the Lesotho Highlands.

Amphibians: Three amphibian species were recorded, two of which are aquatic, namely Drakensberg frog (Amietia delalandii (Duméril \& Bibron, 1841) and Maloti River Frog (Amietia vertebralis (Hewitt, 1927)), as well as one terrestrial Bufonid, called Gariep toad or mountain toad (Vandijkophrynus gariepensis (Smith, 1848)), summarised in Table 6. Ametia delalandii and A. vertebralis are reported as near endemic in the country (NES 2000). A study by LHDA (1998a, 1998b) reported seven amphibian species in both BNR and TNP. These are African clawed frog (Xenopus laevis Daudin, 1802), Gray's stream frog (Strongylopus grayii (Smith, 1849)), Natal ghost frog (Heleophryne natalensis (Hewitt, 1913)) and ranger's toad (Amietophrynus rangeri Hewitt, 1935), as well as the three species recorded in the current study. Generally, Lesotho is reported to have a total of 19 amphibian species, with only two near-endemic species (NES 2000).
Amphibians are reported to be one of the most sensitive group of animals in the world which react rapidly to substantial changes in their environments (Saber et al. 2017). As a result, they are useful indicators of pollution and climate change.

\section{Other biophysical aspects (wetlands)}

Three major wetlands were observed in the core area of the proposed BR, and four others in the transition zone. These wetlands do not only provide habitats for certain flora and fauna species, but also provide water for many river basins in the country as well as transboundary rivers. As a result, these wetlands play a major role in the ecology and hydrology of downstream systems including the provision of water to the Katse Dam, which supplies water to Gauteng province in South Africa. In addition, the wetlands provide water for livestock drinking and domestic use to surrounding communities. However, it was observed that wetlands in the transition zone lack effective management systems and are vulnerable to degradation and consequent reduction of ecosystem services. Some sections within the wetlands are intact while other patches are degrading due to erosion, thus threatening the intact portions. The longterm productivity of these ecosystems is threatened by encroachment through ploughing in surrounding fields and poor road construction practices. In addition, a majority of wetlands in the transition zone are under threat due to cropfield encroachment, roads/paths that go through them, as well as grazing. Similarly, a study

Table 5. A list of recorded reptiles

\begin{tabular}{|c|c|c|c|c|}
\hline Taxon & Common names & $\begin{array}{l}\text { Vernacular } \\
\text { (Sesotho) Names }\end{array}$ & $\begin{array}{l}\text { IUCN Conservation } \\
\text { Status }\end{array}$ & Form \\
\hline Agama atra Peters & Southern rock agama & mankhoshepe & Least Concern & Agama \\
\hline Bitis arietans Merrem & Puff adder & marabe & Not Evaluated & Adder \\
\hline Bitis atropos L. & Berg adder & qooane & Least Concern & Adder \\
\hline Duberria lutrix lutrix Linnaeus & Common slug-eater & & Least Concern & Snake \\
\hline $\begin{array}{l}\text { Hemachatus haemachatus } \\
\text { Bonnaterre }\end{array}$ & Rinkhals & masumo & Least Concern & $\begin{array}{l}\text { Cobra-like } \\
\text { Snake }\end{array}$ \\
\hline $\begin{array}{l}\text { Pseudocordylus melanotus } \\
\text { A. Smith }\end{array}$ & Drakensberg crag lizard & checheiki & Least Concern & Lizard \\
\hline Trachylepis punctatissima Smith & Montane speckled skink & mokholutsoane & Least Concern & Skink \\
\hline Tropidosaura essexi Hewitt & Essex's mountain lizard & & Least Concern & Lizard \\
\hline
\end{tabular}


Table 6. A list of recorded amphibian species

\begin{tabular}{llllll}
\hline Taxon & Common names & $\begin{array}{l}\text { Vernacular } \\
\text { (Sesotho) names }\end{array}$ & Form & Habitat & $\begin{array}{l}\text { IUCN Conservation } \\
\text { Status }\end{array}$ \\
\hline $\begin{array}{l}\text { Amietia delalandii Duméril and } \\
\text { Bibron (= A. dracomontana } \\
\text { Channing) }\end{array}$ & Drakensberg frog & seqaqana & Frog & Aquatic & Least Concern \\
$\begin{array}{l}\text { Amietia vertebralis Hewitt } \\
\text { Vandijkophrynus gariepensis }\end{array}$ & Maluti river frog & letlametlu & Frog & Aquatic & Least Concern \\
A. Smith & $\begin{array}{l}\text { Gariep toad } \\
\text { Mountain toad }\end{array}$ & marokolo & Toad & Terrestrial & Least Concern \\
\hline
\end{tabular}

by Chatanga et al. (2020) indicated that wetlands in the Maloti-Drakensberg region are generally intensively utilised and degraded, with very high anthropogenic pressures, particularly in the highlands of Lesotho. The wetlands are also a habitat for Sloggett's ice rat, which is endemic to southern Africa, being confined to South Africa and Lesotho. However, the species causes damage to the wetlands by digging holes that destroy the vegetation (Figures 3J, K). A study by Mokotjomela et al. (2009) revealed that the damage caused by Sloggett's ice rat through soil erosion and loss exceeds effects of domestic livestock. This mammal species is also found in rocky habitats and alpine grassland, both wet and dry, at elevations of more than $2000 \mathrm{~m}(6600 \mathrm{ft})$. It is reported in the Drakensberg Mountains of the Eastern Cape and KwaZulu-Natal provinces of South Africa, as well as Lesotho (Monadjem et al. 2015).

Notable plant species recorded in the wetlands include: copper wire grass (Merxmuellera macowanii (Stapf) Conert), which is used for making crafts (brooms, baskets, traditional Basotho hats) and thatching, as well as mild rhubarb (Gunnera perpensa L.), water mint (Mentha aquatica L.), and wild clover (Trifolium L. spp), used for medicinal purposes. In addition, Lesotho red-hot poker (Kniphofia caulescens Baker) adds beautiful scenery to high elevation valleys of the Maloti-Drakensberg, particularly in summer (Figure 3E). This plant is endemic to the Maloti-Drakensberg region, even though it is reported to be relatively rare on the South African side (being found in Eastern Cape and KwaZulu-Natal). A study by Chatanga et al. (2019) reported a total of 16 wetland types associated with the Maloti-Drakensberg region, and these include M. macowanii wetland, G. perpensa wetland, K. caulescens wetland and Mentha longifolia-Juncus inflexus wetland. The study also discussed the classification, description and environmental factors of montane wetland vegetation of the Maloti-Drakensberg region.

\section{Conclusion}

The current study provides findings of a rapid biodiversity survey undertaken in Lesotho's first proposed Biosphere Reserve, the core area of which comprises
Bokong Nature Reserve and Tšehlanyane National Park. The survey recorded a total of 380 plant species, 60 of which are endemic to the Drakensberg Mountain Centre (formerly known as Drakensberg Alpine Centre), whereas 30 are declared legally protected in the country. Two plants endemic to Lesotho have been documented in the area namely spiral aloe and Glumicalyx lesuticus. The former is the national flower of Lesotho, which is under immense pressure in the wild due to illegal trade. The near-endemic (confined to South Africa and Lesotho) Drakensberg bamboo was found widespread in Tšehlanyane National Park, and it is reported to provide a habitat for the Red-listed Endangered butterfly species known as Metisella syrinx. However, the search for this butterfly during the survey was not successful.

In the case of fauna, 16 mammalian species were recorded, seven of which are declared legally protected in the country. These include Sloggett's ice rat, which is endemic to southern Africa, being confined to South Africa and Lesotho. This species is however, causing damage to the wetlands, which provide ecosystem services such as water and livestock grazing. These wetlands supply water to the Katse Dam of the Lesotho Highlands Water Project, which in turn provides water to parts of Gauteng in South Africa. A total of 53 bird species were documented, 13 of which are declared legally protected. Among these are the Cape Vulture and Bearded Vulture, which are IUCN listed as Endangered and Near Threatened respectively, and have limited distribution being endemic to southern Africa. Lesotho is currently the main breeding ground for the Bearded Vulture in southern Africa. Two fish species were recorded namely Maloti minnow and rainbow trout, with the former being Lesotho's only known true endemic vertebrate species. A total of seven reptiles were recorded, three of which are venomous snakes namely puff adder, berg adder and rinkhals. In addition, three amphibian species were recorded, two of which are reported as near endemic in the country, namely Amietia delalandii and $A$. vertebralis.

The survey has filled some gaps by providing valuable information on the biodiversity (particularly regarding the flora and avifauna) of the proposed Biosphere Reserve. 
The presence of important species, i.e. some being endemic to Lesotho, endemic to the Maloti-Drakensberg area, Red Data-listed, or declared legally protected in the country, contribute tremendously to the biodiversity value of the proposed BR. Therefore, the findings of the current study will contribute towards justification of the area to be nominated as Lesotho's first Biosphere Reserve under the UNESCO MAB Programme. However, management plans of the proposed Biosphere Reserve should be developed, to address, amongst others, the challenges of managing invasive species and fire that may negatively affect the biodiversity of the area.

\section{Acknowledgements}

The authors are grateful to UNESCO and the Lesotho Government for financial support to undertake the study. We thank the two anonymous reviewers for their useful suggestions to improve the manuscript.

\section{Competing interests}

The authors declare that they have no competing interests.

\section{Authors'contributions}

LSK drafted the manuscript, KK and KM collected the data, and RPS edited the manuscript and added valuable information.

\section{Funding}

The study was financed by UNESCO viz; the UNESCO MAB Programme in Paris, the German Commission for UNESCO and the Lesotho National Commission for UNESCO, as well as the Lesotho Government through the Ministry of Tourism, Environment and Culture (MTEC).

\section{References}

Alexander, G. \& Marais, J., 2008, A Guide to the Reptiles of Southern Africa, Struik Publishers, Cape Town, South Africa.

Barlow, A., Wüster, S., Kelly, C.M.R., Branch, W.R., Phelps, T. \& Tolley, K.A., 2019, 'Ancient habitat shifts and organismal diversification are decoupled in the African viper genus Bitis (Serpentes: Viperidae)', Journal of Biogeography 46, 1234-1248, https://doi.org/10.1111/jbi.13578.

Barnard, K.H., 1938, 'Description of a new species of fresh-water fish from Natal', Annals of the Natal Museum 8(3), 525-528.

Bromilow, C., 2010, Problem plants and alien weeds of South Africa, Briza Publishers, Pretoria, South Africa.

Carbutt, C. \& Edwards, T.J., 2001, 'Cape elements on highaltitude corridors and edaphic islands: historical aspects and preliminary phytogeography', Systematics and Geography of Plants 71, 1033-1061.

Carbutt, C. \& Edwards, T.J., 2004, 'The flora of the Drakensberg Alpine Centre', Edinburgh Journal of Botany 60(3), 581-607, https://doi.org/10.1017/S0960428603000428.

Carbutt, C. \& Edwards, T.J. 2006, 'The endemic and nearendemic angiosperms of the Drakensberg Alpine Centre', South African Journal of Botany 72, 105-132, https://doi. org/10.1016/j.sajb.2005.06.001.

Carbutt, C., 2019, 'The Drakensberg Mountain Centre: A necessary revision of southern Africa's high-elevation centre of plant endemism', South African Journal of Botany 124, 508-529, https://doi.org/10.1016/j.sajb.2019.05.032.

Chatanga, P., Kotze, D.C., Janks, M. \& Sieben, E.J.J., 2019, 'Classification, description and environmental factors of montane wetland vegetation of the Maloti-Drakensberg region and the surrounding areas', South African Journal of Botany 125, 221-233, https://doi.org/10.1016/j. sajb.2019.04.028.
Chatanga, P., Kotze, D.C., Okello, T.W. \& Sieben, E.J., 2020, 'Ecosystem services of high-altitude Afromontane palustrine wetlands in Lesotho', Ecosystem Services 45, 101185, https://doi.org/10.1016/j.ecoser.2020.101185.

Colahan, B.D. 2004, 'The status and conservation of vultures in the Free State Province of South Africa' in Monadjem, A., Anderson, M.D., Piper, S.E. \& Boshoff, A.F (eds.), Vultures in The Vultures of Southern Africa - Quo Vadis?, Proceedings of a workshop on vulture research and conservation in southern Africa., Birds of Prey Working Group, Johannesburg.

Cooper-Driver, G., Letšela, T. \& Polaki, M., 2008, Searching for ferns in Lesotho, Ashworth Publishing Co., Cornwall, UK.

Cowling, R.M. \& Hilton-Taylor, C., 1994, 'Patterns of Plant Diversity and Endemism in Southern Africa: An overview' in Huntley, B.J. (ed.), Botanical Diversity in Southern Africa, 31-52, National Botanical Institute, Pretoria.

Di Vittorio, M., Hema, E.M., Dendi, D., Akani, G.C., Cortone, G., López-López, P., Amadi, N., Hoinsoudé Ségniagbeto, G., Battisti, C. \& Luiselli, L., 2018, 'The conservation status of West African vultures: an updated review and a strategy for conservation', Life and Environment 68 (1), 33-43.

Donázar, J.A., Hiraldo, F. \& Bustamante, J., 1993, 'Factors influencing nest site selection, breeding density and breeding success in the bearded vulture (Gypaetus barbatus)', Journal of Applied Ecology 504-514.

Du Preez, L. \& Carruthers, V., 2009, A complete guide to the frogs of Southern Africa, Struik Nature, Cape Town, South Africa.

Grab, S.W. \& Nash, D.J., 2020, " "But what silence! No more gazelles..." occurrence and extinction of fauna in Lesotho, southern Africa, since the late Pleistocene', Quaternary International. 
Hilliard, O.M. \& Burtt, B.L., 1987, The Botany of the Southern Natal Drakensberg, Annals of the Kirstenbosch Botanical Garden, 15, 253.

Hockey, P,A,R,, Dean, W,R,J, \& Ryan, P.G., (eds.), 2006, Roberts birds of Southern Africa ( $7^{\text {th }}$ edn), Russel Friedman Books, Halfway House, South Africa.

IUCN 2018, 'The IUCN Red Data List of Threatened Species, Version 2018-2', viewed 1 October 2018, http://www. iucnredlist.org

Jacot Guillarmod, A., 1971, Flora of Lesotho, Cramer, Lehre.

Jubb, RA 1971, 'Oreodaimon quathlambae (Labeo quathlambae Barnard) in the Tsoelikana river, south-west Lesotho', Piscator 81, 39-42.

Kobisi, K., 2005, Preliminary checklist of the plants of Lesotho, Southern African Botanical Diversity Network Report no. 34, SABONET, Pretoria \& Roma, ISBN 1-919795-67-7.

Kobisi, K, \& Kose, L.E., 2003, A checklist of Lesotho grasses, Southern Africa Botanical Diversity Network (SABONET) Report no. 17, Pretoria, ISBN 1-919795-68-5.

Kobisi, K., Seleteng-Kose, L. \& Moteetee, A., 2019, 'Invasive alien plants occurring in Lesotho: their ethnobotany, potential risks, distribution and origin', Bothalia 49(1), https:// doi.org/10.4102/abc.v49i1.2453.

Kubheka, P.S., Chakona, A. \& Magunzula, D.N., 2017, 'The Maloti minnow Pseudobarbus quathlambae (Barnard, 1938) is not extinct in South Africa', African Journal of Aquatic Science 42(3), 303-306, https://doi.org/10.2989/1 6085914.2017 .1363705$.

Lahmeyer Macdonald Consortium \& Oliver Shand Consortium 1986, Lesotho Highlands Water Project, Kingdom of Lesotho, Ministry of Water, Energy and Mining.

Letšela, T., Witkowski, E.T.F. \& Balkwill, K., 2003, 'Plant resources used for subsistence in Tšehlanyane and Bokong in Lesotho', Economic Botany 57(4), 619-639.

Lesotho Highlands Development Authority (LHDA) 1998a, Conservation development and management plan for Bokong Nature Reserve, LHDA Contract 604.

Lesotho Highlands Development Authority (LHDA) 1998b, Conservation development and management plan for Tšehlanyane National Park, LHDA Contract 604.

May, E.D., 2000, The Indigenous forests of Lesotho, Morija Printing Works, Morija, Lesotho.

Meter, E.B., Edwards, T.J., Rennie, M.A. \& Granger, J.E., 2002, 'A checklist of the plants of Mahwaqa Mountain, KwaZuluNatal', Bothalia 32(1), 101-115.

Ministry of Tourism, Environment and Culture (MTEC) Legal Notice No. 36 of 1969, as amended by Legal Notice No. 93 of 2004 and No.38 of 2006

Moffett, R., 1997, Grasses of the Eastern Free State, their description and uses, UNIQWA, Phuthaditjhaba.

Moffett, R., 2010, Sesotho plant and animal names and plants used by the Basotho, Sun Press, Bloemfontein, South Africa.

Mokotjomela, T., Schwaibold, U. \& Pillay, N., 2009, 'Does the ice rat Otomys sloggetti robertsi contribute to habitat change in Lesotho?' Acta Oecologica 35, 437-443, https:// doi.org/10.1016/j.actao.2009.01.004.

Monadjem, A., Taylor, P.J., Denys, C. \& Cotterill, F.P.D., 2015, Rodents of Sub-Saharan Africa: A biogeographic and taxonomic synthesis, De Gruyter, Berlin, Germany.

Mucina, L. \& Rutherford, M.C. (eds.), 2006, The vegetation of South Africa, Lesotho and Swaziland, Strelitzia vol. 19, South African Biodiversity Institute, Pretoria.
Mucina, L., Hoare, D.B., Lötter, M.C., Du Preez, J., Rutherford, M.C., Scott-Shaw, C.R., Bredenkamp, G.J., Powrie, L.W., Scott, L., Camp, K.G.T., Cilliers, S.S., Bezuidenhout, H., Mostert, T.H., Siebert, S.J., Winter, P.J.D., Burrows, J.E., Dobson, L., Ward, R.A., Stalmans, M., Oliver, E.G.H., Siebert, F., Schmidt, E., Kobisi, K. \& Kose, L., 2006, Grassland Biome in L. Mucina \& M.C. Rutherford (eds), The Vegetation of South Africa, Lesotho and Swaziland, Strelitzia 19, South African National Biodiversity Institute, Pretoria.

National Environment Secretariat (NES) 2000, Biological diversity in Lesotho: a country study, Morija Printing Works Maseru, Lesotho.

Ogada, D.L., Keesing, F. \& Virani, M.Z., 2012, 'Dropping dead: causes and consequences of vulture population declines worldwide', Annals of the New York Academy of Sciences 1249, 57-71.

Phillips, E.P., 1917, 'A contribution to the flora of the Leribe Plateau and environs', Annals of the South African Museum $16,1-379$.

Pooley, E., 1993, The Complete field guide to trees of Natal, Zululand \& Transkei, Natal Flora Publications Trust, Durban, South Africa.

Pooley, E 1998, A Field guide to wild flowers of KwaZuluNatal and the eastern region, Natal Flora Publication Trust, Durban, South Africa.

Pooley, E., 2003, Mountain flowers: A field guide to flora of Drakensberg and Lesotho, Natal Flora publications Trust, Durban, South Africa.

Porter, R., Scott-Shaw, C.R. \& Thomson, P., 1999, Nomination proposal for the Drakensberg Park to be listed as a World Heritage Site, KwaZulu-Natal Nature Conservation Service, Pietermaritzburg.

Saber, S., Tito, W., Said, R., Mengistou, S. \& Alqahtani, A., 2017, Amphibians as bioindicators of the health of some wetlands in Ethiopia', The Egyptian Journal of Hospital Medicine 66 (1), 66-73.

Schmitz, M.O., 1982, Wild flowers of Lesotho, ESSA, Roma, Lesotho.

Sinclair, I, Hockey, P, \& Tarboton, W., 2002, Birds of southern Africa: the region's most comprehensively illustrated guide, Struik Publishers, Cape Town, South Africa.

Soderstrom, T.R. \& Ellis, R.P., 1982, 'Taxonomic status of the endemic South African bamboo, Thamnocalamus tessellatus', Bothalia 14(1), 53-67.

Stuart, C. \& Stuart, M., 2015, Field guide to mammals of Southern Africa, Struik Publishers, Cape Town, South Africa.

Talukdar, S., 2002, 'Lesotho Plant Red Data List' in Golding, J. (ed.), Southern African Plant Red Data Lists, Report No. 14, Southern African Botanical Diversity Network, Pretoria, South Africa.

UNESCO, 1996, Biosphere reserves: the Seville Strategy and the Statutory Framework of the World Network, UNESCO, Paris.

Van Oudtshoorn, F., 1999, Guide to grasses of Southern Africa, Briza Publications, Pretoria, South Africa.

Van Wyk, B-E. \& Gericke, N., 2000, People's plants. A guide to useful plants of Southern Africa, Briza Publications, Pretoria, South Africa.

Van Wyk, A.E. \& Smith, G.F., 2001, Regions of floristic endemism in Southern Africa, Umdaus Press, Pretoria, South Africa.

Van Wyk, B-E. \& Smith, G., 2014, Guide to the Aloes of South Africa, Briza Publications, Pretoria, South Africa. 
Van Wyk, B-E. \& Van Wyk, P., 1997, Field guide to trees of Southern Africa, Struik Publishers, Cape Town, South Africa. Van Wyk, B-E., Van Oudtshoorn, B. \& Gericke, N., 2009, Medicinal plants of South Africa $2^{\text {nd }}$ Edn, Briza Publications, Pretoria, South Africa.

Wybenga, W., 2006, 'Wildflowers of Lesotho's Tšehlanyane National Park', Veld \& Flora 92(2), 81-85.

\section{Web material}

http://redlist.sanbi.org (viewed 20 September 2020) https://www.iucnredlist.org (viewed 26 September 2020) http://pza.sanbi.org/ (viewed 22 September 2020) 